\title{
Article \\ Branched-Chain Amino Acids Can Predict Mortality in ICU Sepsis Patients
}

\author{
Alexander Christian Reisinger ${ }^{1}{ }^{(D}$, Florian Posch ${ }^{2}$, Gerald Hackl ${ }^{1}$, Gunther Marsche $\left.{ }^{3} \mathbb{(}\right)$, Harald Sourij ${ }^{4}(\mathbb{D}$, \\ Benjamin Bourgeois ${ }^{5}$, Kathrin Eller ${ }^{6}\left[\mathbb{D}\right.$, Tobias Madl ${ }^{5,7, *}$ ) and Philipp Eller ${ }^{1, *}$ (])
}

1 Intensive Care Unit, Department of Internal Medicine, Medical University of Graz, 8036 Graz, Austria; alexander.reisinger@medunigraz.at (A.C.R.); gerald.hackl@medunigraz.at (G.H.)

2 Department of Internal Medicine, Division of Oncology, Medical University of Graz, 8036 Graz, Austria; florian.posch@medunigraz.at

3 Otto Loewi Research Center for Vascular Biology, Immunology and Inflammation, Division of Pharmacology, Medical University of Graz, 8036 Graz, Austria; gunther.marsche@medunigraz.at

4 Department of Internal Medicine, Division of Endocrinology and Diabetology, Medical University of Graz, 8036 Graz, Austria; ha.sourij@medunigraz.at

5 Gottfried Schatz Research Center for Cell Signaling, Metabolism and Aging, Molecular Biology and Biochemistry, Medical University of Graz, 8036 Graz, Austria; benjamin.bourgeois@medunigraz.at

6 Department of Internal Medicine, Division of Nephrology, Medical University of Graz, 8036 Graz, Austria; kathrin.eller@medunigraz.at

7 BioTechMed Graz, 8036 Graz, Austria

* Correspondence: tobias.madl@medunigraz.at (T.M.); philipp.eller@medunigraz.at (P.E.); Tel.: +43-316-385-82215 (P.E.); Fax: +43-316-385-17527 (P.E.)

Citation: Reisinger, A.C.; Posch, F.; Hackl, G.; Marsche, G.; Sourij, H.; Bourgeois, B.; Eller, K.; Madl, T.; Eller, P. Branched-Chain Amino Acids Can Predict Mortality in ICU Sepsis Patients. Nutrients 2021, 13, 3106. https://doi.org/10.3390/nu13093106

Academic Editor: Gary

David Lopaschuk

Received: 5 August 2021

Accepted: 1 September 2021

Published: 3 September 2021

Publisher's Note: MDPI stays neutral with regard to jurisdictional claims in published maps and institutional affiliations.

Copyright: (c) 2021 by the authors. Licensee MDPI, Basel, Switzerland. This article is an open access article distributed under the terms and conditions of the Creative Commons Attribution (CC BY) license (https:// creativecommons.org/licenses/by/ $4.0 /)$.
Abstract: Sepsis biomarkers and potential therapeutic targets are urgently needed. With proton nuclear magnetic resonance $\left({ }^{1} \mathrm{H}\right.$ NMR) spectroscopy, several metabolites can be assessed simultaneously. Fifty-three adult medical ICU sepsis patients and 25 ICU controls without sepsis were prospectively enrolled. ${ }^{1} \mathrm{H}$ NMR differences between groups and associations with 28-day and ICU mortality were investigated. In multivariate metabolomic analyses, we found separate clustering of ICU controls and sepsis patients, as well as septic shock survivors and non-survivors. Lipoproteins were significantly different between sepsis and control patients. Levels of the branched-chain amino acids (BCAA) valine (median 43.3 [29.0-53.7] vs. 64.3 [47.7-72.3] normalized signal intensity units; $p=0.005)$, leucine (57.0 [38.4-71.0] vs. 73.0 [54.3-86.3]; $p=0.034$ ) and isoleucine (15.2 [10.9-21.6] vs. $17.9[16.1-24.4] ; p=0.048$ ) were lower in patients with septic shock compared to those without. Similarly, BCAA were lower in ICU non-survivors compared to survivors, and BCAA were good discriminators for ICU and 28-day mortality. In uni- and multivariable logistic regression analyses, higher BCAA levels were associated with decreased ICU- and 28-day mortality. In conclusion, metabolomics using ${ }^{1} \mathrm{H}$ NMR spectroscopy showed encouraging potential for personalized medicine in sepsis. BCAA was significantly lower in sepsis non-survivors and may be used as early biomarkers for outcome prediction.

Keywords: sepsis; ICU; metabolomics; NMR spectroscopy; branched-chain amino acids; lipoproteins

\section{Introduction}

Sepsis is a common global life-threatening medical condition, and more than 31 million patients each year suffer from sepsis or septic shock [1]. The absolute number of sepsis patients is continuously increasing over the last years because of a more widespread use of immunosuppressive therapies, increasing age and the emerge of multi-resistant bacteria [2]. Sepsis is not simply the presence of bacteremia, but also triggered by the host's response to the infection causing organ dysfunction. This concept is reflected in the current sepsis-3 definition [3]. The diagnosis of sepsis includes a suspected infection and the presence of a new organ dysfunction measured by the sequential organ failure assessment (SOFA) 
score. Septic shock represents a sicker subpopulation with an increased blood lactate level above $2 \mathrm{mmol} / \mathrm{L}$ and the need for vasopressor therapy to maintain a mean arterial pressure (MAP) over $65 \mathrm{mmHg}$. Apart from lactate, other biomarkers may be helpful in the early recognition and diagnosis of sepsis, in the assessment of disease severity, and for outcome prediction. Some inflammatory markers such as C-reactive protein (CRP) and procalcitonin (PCT) were not consistently able to predict the outcome of sepsis patients, and therefore better biomarkers are needed [4]. We were recently able to show that lipoprotein functionality parameters such as the arylesterase activity of high-density lipoprotein (HDL) associated paraoxonase 1 may be useful for outcome prediction in sepsis [5]. Therefore, we aimed to validate and extend these findings using proton nuclear magnetic resonance ( ${ }^{1} \mathrm{H}$ NMR) spectroscopy. This emerging technique within the field of medicine, enables detection, identification, and quantification of metabolites and lipoproteins in serum or plasma samples with exceptionally good reproducibility and robustness [6,7]. ${ }^{1} \mathrm{H}$ NMR can be used to assess samples for several metabolites simultaneously, providing the socalled metabolomic profile or metabolome. The advantage of this evolving method is the option of either a targeted or an untargeted approach ("global metabolomics"), making the identification of metabolites that were unknown to play a role in the investigated disease possible [8]. Furthermore, changes within the genome, transcriptome and the proteome can be displayed within the metabolome, thus providing a direct read-out for the functional alterations associated therewith.

In this study, we applied metabolomic methods using ${ }^{1} \mathrm{H}$ NMR spectroscopy to analyze metabolites and lipoprotein-derived parameters from patients with sepsis and septic shock admitted to the intensive care unit (ICU) as well as ICU patients (controls) without sepsis or bacteremia to identify biomarkers associated with ICU outcome.

\section{Materials and Methods}

\subsection{Study Population and Study Design}

We recruited patients older than 18 years with sepsis and septic shock and ICU controls admitted to the ICU of the Department of Internal Medicine at the Medical University of Graz, Austria, as formerly published [5]. In brief, sepsis-3-criteria were applied to define sepsis patients [3], and the control group consisted of consecutive ICU patients without sepsis or bacteremia at the time of sampling. The study protocol was approved by the Institutional Review Board (IRB) of the Medical University of Graz, Austria (30-258 ex $17 / 18$ ) and complied with the Declaration of Helsinki. Written informed consent was obtained from all conscious participants. In comatose non-survivors, the IRB waived the need for written informed consent.

\subsection{Laboratory Analyses}

Blood cell count, serum creatinine, bilirubin, CRP, PCT, and interleukin-6 (IL-6) were measured using a Sysmex (Sysmex Austria GmbH, Vienna, Austria) or Cobas (Roche Diagnostics, Mannheim, Germany) analyzer as appropriate. For further analyses, we centrifuged peripheral blood samples at $3000 \mathrm{rpm}$ for $10 \mathrm{~min}$ and stored aliquots at $-80^{\circ} \mathrm{C}$ without any thawing/freezing cycles. Metabolomic analyses were performed in batch to avoid any inter-assay variance.

\subsection{Reagents}

Sodium phosphate, dibasic $\left(\mathrm{Na}_{2} \mathrm{HPO}_{4}\right)$, sodium hydroxide $(\mathrm{NaOH})$, hydrochloric acid $(\mathrm{HCl}, 32 \% \mathrm{~m} / \mathrm{v})$, and sodium azide $\left(\mathrm{NaN}_{3}\right)$ were obtained from VWR International (Darmstadt, Germany). 3(Trimethylsilyl) propionic acid-2,2,3,3-d $\mathrm{d}_{4}$ sodium salt (TSP) was obtained from Alfa Aesar (Karlsruhe, Germany). Deuterium oxide $\left(\mathrm{D}_{2} \mathrm{O}\right)$ was obtained from Cambridge Isotopes Laboratories (Tewksbury, MA, USA). Deionized water was purified using an in-house Milli-Q Advantage Water Purification System from Millipore (Schwalbach, Germany). All chemicals were used without further purification. The phosphate NMR buffer solution was prepared by dissolving $5.56 \mathrm{~g}$ of anhydrous $\mathrm{Na}_{2} \mathrm{HPO}_{4}, 0.4 \mathrm{~g}$ of TSP, 
and $0.2 \mathrm{~g} \mathrm{NaN}_{3}$, in $400 \mathrm{~mL}$ of $\mathrm{D}_{2} \mathrm{O}$ and adjusted to $\mathrm{pH} 7.4$ with $1 \mathrm{M} \mathrm{NaOH}$ and $\mathrm{HCl}$. Upon addition of $\mathrm{D}_{2} \mathrm{O}$ to a final volume of $500 \mathrm{~mL}$ the $\mathrm{pH}$ was re-adjusted to $\mathrm{pH} 7.4$ with $1 \mathrm{M}$ $\mathrm{NaOH}$ and $\mathrm{HCl}$.

\subsection{Lipoprotein Quantification Using NMR}

Blood serum lipoproteins were analyzed on a Bruker $600 \mathrm{MHz}$ Avance Neo NMR spectrometer using the Bruker IVDr lipoprotein subclass analysis protocol. Serum samples were thawed, and $330 \mu \mathrm{L}$ of each sample mixed with $330 \mu \mathrm{L}$ of Bruker serum buffer (Bruker, Rheinstetten, Germany). The samples were mixed gently, and $600 \mu \mathrm{L}$ of the mixed sample were transferred into a $5 \mathrm{~mm}$ SampleJet rack tube (Bruker, Rheinstetten, Germany). Proton spectra were obtained at a constant temperature of $310 \mathrm{~K}$ using a standard nuclear Overhauser effect spectroscopy (NOESY) pulse sequence (Bruker: noesygppr1d), a CarrPurcedll-Meiboom-Gill (CPMG) pulse sequence with presaturation during the relaxation delay (Bruker: cpmgpr1d) to achieve water suppression, and a standard 2D J-resolved (JRES) pulse sequence (Bruker: jresgpprqf) [9]. Data analysis was carried out using the Bruker IVDr Lipoprotein Subclass Analysis (B.I.LISA ${ }^{\mathrm{TM}}$ ) method.

\subsection{Metabolic Quantification Using NMR}

To remove proteins and to quench enzymatic reactions in the samples, $200 \mu \mathrm{L}$ serum was mixed with $400 \mu \mathrm{L}$ methanol and stored at $-20^{\circ} \mathrm{C}$ for $1 \mathrm{~h}$ until further processing. Afterwards the samples were spun at $17,949 \mathrm{rcf}$ at $4{ }^{\circ} \mathrm{C}$ for $30 \mathrm{~min}$. Supernatants were lyophilized and $500 \mu \mathrm{L}$ of NMR buffer in $\mathrm{D}_{2} \mathrm{O}$ were added to the samples and transferred to $5 \mathrm{~mm}$ NMR tubes. All NMR experiments were performed at $310 \mathrm{~K}$ on an AVANCE ${ }^{\mathrm{TM}} \mathrm{Neo}$ Bruker Ultrashield $600 \mathrm{MHz}$ spectrometer equipped with a TXI probe head and processed as described previously [10]. The 1D CPMG (Carr-Purcell_Meiboom_Gill) pulse sequence (cpmgpr1d, 512 scans, 73,728 points in F1, 11,904.76 HZ spectral width, 512 transients, recycle delays $4 \mathrm{~s}$ ) with water suppression using pre-saturation, was used for ${ }^{1} \mathrm{H} 1 \mathrm{D}$ NMR experiments. Bruker Topspin version 4.0.2 was used for NMR data acquisition. The spectra for all samples were automatically processed (exponential line broadening of $0.3 \mathrm{~Hz}$ ), phased, and referenced using TSP at $0.0 \mathrm{ppm}$ using Bruker Topspin 4.0.2 software (Bruker $\mathrm{GmbH}$, Rheinstetten, Germany). Spectra pre-processing and data analysis was carried out using the state-of-the-art data analysis pipeline proposed by the group of Prof. Jeremy Nicholson at Imperials College London using Matlab ${ }^{\circledR}$ scripts and MetaboAnalyst 4.0 [11]. NMR data were imported to Matlab ${ }^{\circledR}$ vR2014a (Mathworks, Natick, MA, USA), regions around the water, TSP, and remaining methanol signals excluded, and probabilistic quotient normalization [12] was performed to correct for sample metabolite dilution; and reported concentrations corresponded to normalized concentrations.

\subsection{Statistical Analyses}

To identify changes in lipoprotein and metabolic profiles, multivariate statistical analysis was performed as described previously [13], and included principal component analysis (PCA), partial least squares-discriminant analysis (PLS-DA), orthogonal-partial least squares-discriminant analysis (O-PLS-DA) [14], with associated data consistency checks and 7-fold cross-validation, expressed by $\mathrm{Q}^{2}$. For the PCA and O-PLS-DA, NMR data were analyzed in Matlab ${ }^{\circledR}$ vR2014a, and figures were prepared using MetaboAnalyst [11]. All other statistical analyses were performed with GraphPad Prism 8.0 (GraphPad Software, San Diego, CA, USA), SPSS 26 (SPSS Inc., Chicago, IL, USA), and Stata 15.0 (Stata Corp., Houston, TX, USA). Continuous variables were summarized as medians (25th-75th percentile), and categorical variables as absolute frequencies (\%). Associations between variables were computed with cross-tabulations, Mann-Whitney U-tests, $\chi^{2}$-tests, and Fisher's exact tests, as appropriate. Spearman's rank-based correlation coefficient was used for correlation analyses. Formal adjustment for multiple testing was performed with the Sidak correction method. The prognostic associations between 28-day as well as ICU mortality and other potential baseline predictors were computed with univariable and 
multivariable logistic regression. Longitudinal trajectories of biomarker variables were analyzed with linear mixed models.

\section{Results}

\subsection{Baseline Characteristics and Laboratory Results of the Study Population}

In our study, 53 patients in the sepsis and 25 patients in the control cohort were included (Table 1). The median age of sepsis patients was 66 years (50-75), whereas ICU-survivors were 63 (46-76) and non-survivors 66 (53-73) years of age $(p=0.86)$, as previously reported [5]. In brief, females accounted for $40 \%$ of the sepsis study population and most infections (91\%) were community-acquired. The primary focus of infection was the lung with $42 \%$, followed by the abdomen with $17 \%$ and the urinary tract with $11 \%$. Three, eight, and ten patients had received propofol, insulin, and glucose before sample acquisition, respectively. Parenteral nutrition was present in five patients at the time of sample acquisition, while zero patients had enteral nutrition. Samples were obtained in a median $3.3 \mathrm{~h}$ after ICU admission.

Table 1. Demographics, disease severity, and patient outcomes in the sepsis and control cohort.

\begin{tabular}{|c|c|c|c|}
\hline Variables & Sepsis Patients $(\mathrm{N}=53)$ & Controls $(\mathrm{N}=25)$ & $p$-Value \\
\hline \multicolumn{4}{|c|}{ Demographics \& Premedication } \\
\hline Age (years) & $66(50-75)$ & $72(65-79)$ & 0.012 \\
\hline Female sex & $21(40 \%)$ & $15(60 \%)$ & 0.144 \\
\hline Anti-diabetic medication & $12(23 \%)$ & $8(32 \%)$ & 0.413 \\
\hline Pre-existing diabetes & $15(28 \%)$ & $8(32 \%)$ & 0.793 \\
\hline Pre-existing liver disease & $3(6 \%)$ & $2(8 \%)$ & 0.653 \\
\hline \multicolumn{4}{|l|}{$\begin{array}{c}\text { Disease severity and patient } \\
\text { outcomes }\end{array}$} \\
\hline SOFA score (points) & $9(7-13)$ & $5(3-9)$ & $<0.0001$ \\
\hline Presence of shock & $26(49 \%) \#$ & $7(28 \%) *$ & 0.079 \\
\hline 28-day mortality & $25(47 \%)$ & $4(16 \%)$ & 0.011 \\
\hline ICU mortality & $19(36 \%)$ & $4(16 \%)$ & 0.110 \\
\hline
\end{tabular}

SOFA = sequential organ failure assessment, ICU = intensive care unit. ${ }^{*}$ Necessity of vasopressors and lactate $>2 \mathrm{mmol} / \mathrm{l}$. \# according to sepsis-3 definition. Please also refer to Reisinger et al. Front Med.2020 [5].

The baseline characteristics between sepsis ICU-survivors and non-survivors were mostly similar, but patients with septic shock compared to those without shock had higher PCT levels, higher serum creatinine levels, and higher SOFA scores, indicating higher disease severity. The ICU- and 28-day mortality of the sepsis cohort were 36 and $47 \%$, respectively. In the ICU control cohort, e.g., patients with acute cardiovascular disease, cardiac arrest, intoxications, acute kidney injury, and other conditions, but without sepsis or bacteremia, the median age was 72 (65-79) years ( $p=0.01$ compared to sepsis cohort) with $60 \%$ being female. Despite being slightly older, ICU controls had similar rates of pre-existing diabetes or liver disease. Inflammatory markers including white blood count (WBC), CRP, and PCT were lower in ICU controls compared to sepsis patients.

\subsection{Targeted Metabolomic Assessment of Lipoproteins in the Sepsis and Control Cohort}

In a first step, targeted metabolomic assessment of lipoproteins using ${ }^{1} \mathrm{H}$ NMR spectroscopy was performed to confirm and extend our previous results [5]. One patient in the sepsis cohort had to be excluded before lipoprotein analyses were performed due to insufficient sample volume in the aliquot. In the multivariate data analyses using PCA comparing the metabolomic lipoprotein profiles, we found differences between the sepsis and control group with a principal component 1 and 2 (PC1 and PC2) of $76.8 \%$ and $11.0 \%$, respectively (Figure 1A). O-PLS-DA showed a separate clustering for sepsis and ICU con- 
trol patients with a strong-moderate goodness of fit (correlation coefficient $R^{2} Y=0.405$ ) and a cross-validation score $\mathrm{Q}^{2}$ of $0.292(p<0.01$, Figure $1 \mathrm{~A})$. Several lipoproteins were significantly different between the sepsis and the ICU control cohort (Table 2, Supplementary Table S1). In detail, patients in the sepsis compared to the ICU control group had, among other variables, significantly lower levels of HDL free cholesterol (HDFC), HDL cholesterol, and higher levels of very-low-density lipoprotein (VLDL) parameters (Figure 1B,C). In univariable logistic regression analyses for 28-day and ICU mortality, levels of most lipoproteins were not statistically significantly associated with outcomes (Supplementary Tables S2 and S3).
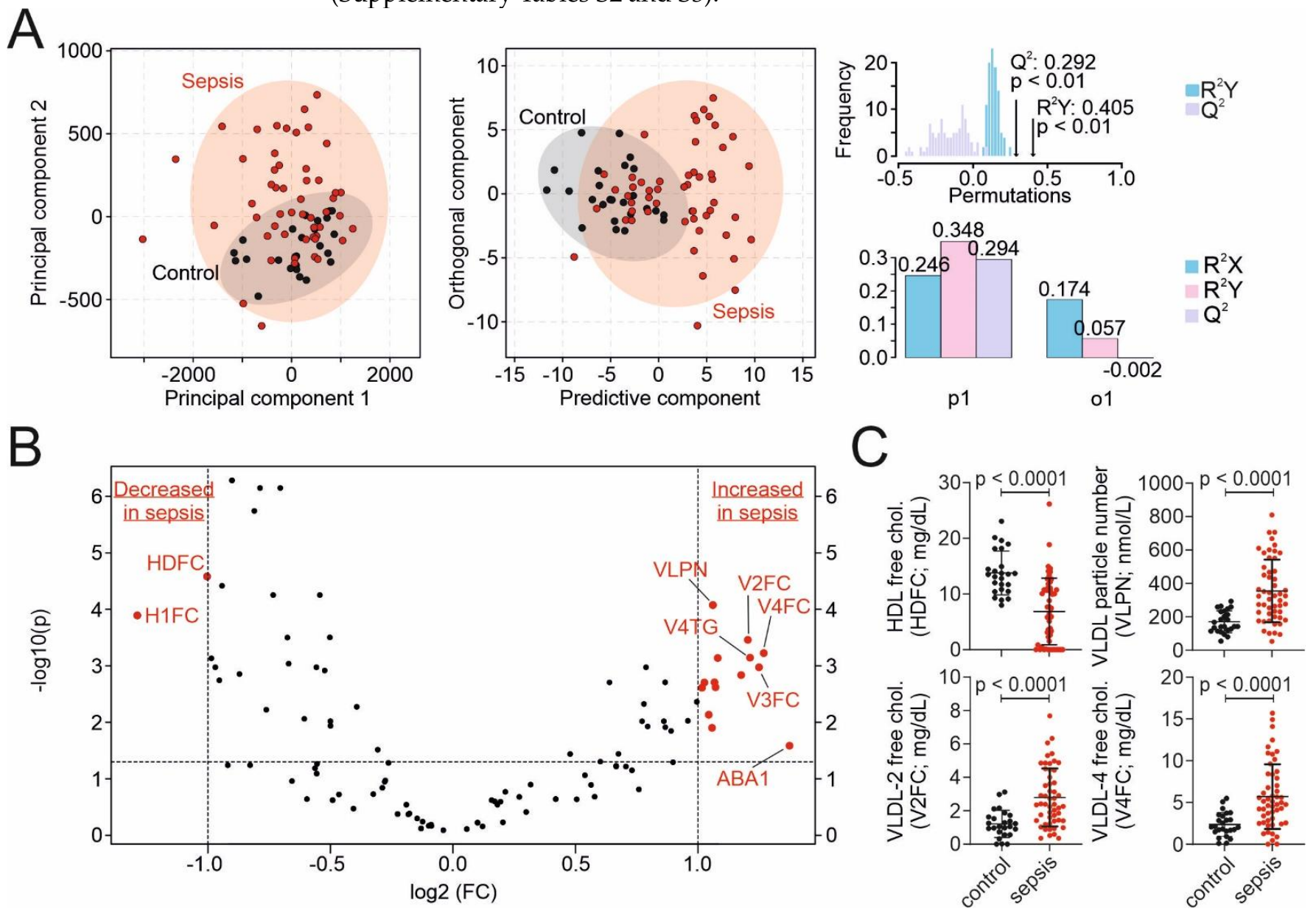

Figure 1. Targeted metabolomic assessment of lipoproteins. (A) Multivariate data analyses of lipoprotein parameters with principal component analyses (PCA) and orthogonal partial least squares discriminant analysis (O-PLS-DA) for the differentiation between ICU controls (black) and sepsis patients (red). PCA: principal component (PC) 1 of $76.8 \%$ and PC2 of $11.0 \%$. O-PLS-DA: correlation coefficient $\mathrm{R}^{2} \mathrm{Y}=0.405$ and cross-validation score $\mathrm{Q}^{2}$ of $0.292(p<0.01)$. (B) Differences in lipoproteins between sepsis and control patients on a logarithmic scale (volcano plot). The vertical dashed lines mark the border to two-fold changes. The horizontal dashed line marks the level of significance at 0.05. (C) Boxplots of the most significant and most changed lipoproteins of sepsis (red color) and control (black color) patients. Abbreviations: HDFC = high density free cholesterol; H1FC = HDL-1 free cholesterol; VLDL = very-low-density lipoprotein; VLPN = VLDL particle number, V2FC = VLDL-2 free cholesterol; V3FC = VLDL-3 free cholesterol; V4FC = VLDL-4 free cholesterol; V4TG = VLDL-4 triglycerides; ABA1 = apolipoprotein B100 to apolipoprotein A-I ratio.

Table 2. Lipid parameters in ICU sepsis patients and ICU controls assessed by targeted metabolomic analyses.

\begin{tabular}{ccccc}
\hline Variables & Sepsis Patients $(\mathbf{N}=\mathbf{5 2})$ & Controls $(\mathbf{N}=\mathbf{2 5})$ & $p$-Value & Below Sidak-Threshold * \\
\hline Main classes & & & & \\
\hline Triglycerides $(\mathrm{mg} / \mathrm{dL})$ & $185(129-310)$ & $101(87-157)$ & $<0.001$ & yes \\
\hline Total cholesterol $(\mathrm{mg} / \mathrm{dL})$ & $117(106-148)$ & $143(119-194)$ & 0.011 & no \\
\hline LDL cholesterol $(\mathrm{mg} / \mathrm{dL})$ & $57(39-76)$ & $77(53-106)$ & 0.012 & no \\
\hline
\end{tabular}


Table 2. Cont.

\begin{tabular}{|c|c|c|c|c|}
\hline Variables & Sepsis Patients $(N=52)$ & Controls $(\mathrm{N}=25)$ & $p$-Value & Below Sidak-Threshold * \\
\hline HDL cholesterol (mg/dL) & $20(13-30)$ & $41(32-51)$ & $<0.001$ & yes \\
\hline Total ApoA1 (mg/dL) & $72(52-98)$ & $120(98-139)$ & $<0.001$ & yes \\
\hline Total ApoA2 (mg/dL) & $19(15-23)$ & $24(20-27)$ & $<0.001$ & no \\
\hline Total ApoB100 (mg/dL) & $82(63-103)$ & $74(59-87)$ & 0.171 & no \\
\hline LDL to HDL ratio & $2.6(1.7-3.7)$ & $1.9(1.5-2.5)$ & 0.009 & no \\
\hline ApoB100 to ApoA1 ratio & $1.3(0.7-1.7)$ & $0.6(0.5-0.8)$ & $<0.001$ & yes \\
\hline \multicolumn{5}{|l|}{ Particles } \\
\hline $\begin{array}{l}\text { Total particle number } \\
\text { (nmol/L) }\end{array}$ & 1494 (1149-1877) & 1338 (1063-1588) & 0.171 & no \\
\hline $\begin{array}{l}\text { VLDL particle number } \\
(\mathrm{nmol} / \mathrm{L})\end{array}$ & $324(205-490)$ & $142(118-233)$ & $<0.001$ & yes \\
\hline $\begin{array}{l}\text { IDL particle number } \\
(\mathrm{nmol} / \mathrm{L})\end{array}$ & $157(79-300)$ & $87(61-137)$ & 0.002 & no \\
\hline $\begin{array}{l}\text { LDL particle number } \\
\text { (nmol/L) }\end{array}$ & $930(737-1225)$ & $1028(720-1254)$ & 0.640 & no \\
\hline \multicolumn{5}{|l|}{$\begin{array}{l}\text { Triglycerides in } \\
\text { subclasses }\end{array}$} \\
\hline VLDL (mg/dL) & $94(63-199)$ & $50(43-111)$ & 0.010 & no \\
\hline IDL (mg/dL) & $13(7-31)$ & $6(3-15)$ & 0.007 & no \\
\hline $\mathrm{LDL}(\mathrm{mg} / \mathrm{dL})$ & $32(19-58)$ & $22(17-30)$ & 0.006 & no \\
\hline $\mathrm{HDL}(\mathrm{mg} / \mathrm{dL})$ & $15(10-19)$ & $13(10-16)$ & 0.124 & no \\
\hline \multicolumn{5}{|l|}{ Cholesterol in subclasses } \\
\hline VLDL (mg/dL) & $28(20-41)$ & $17(12-24)]$ & $<0.001$ & no \\
\hline IDL (mg/dL) & $21(11-37)$ & $11(7-16)$ & 0.002 & no \\
\hline $\mathrm{LDL}(\mathrm{mg} / \mathrm{dL})$ & $57(39-76)$ & $77(53-106)$ & 0.012 & no \\
\hline $\mathrm{HDL}(\mathrm{mg} / \mathrm{dL})$ & $20(13-30)$ & $41(32-51)$ & $<0.001$ & yes \\
\hline \multicolumn{5}{|l|}{$\begin{array}{c}\text { Free cholesterol in } \\
\text { subclasses }\end{array}$} \\
\hline VLDL (mg/dL) & $13(10-20)$ & $8(6-13)$ & 0.002 & no \\
\hline IDL $(\mathrm{mg} / \mathrm{dL})$ & $6(3-11)$ & $3(2-4)$ & $<0.001$ & no \\
\hline LDL (mg/dL) & $24(19-34)$ & $29(21-39)$ & 0.107 & no \\
\hline HDL (mg/dL) & $6(1-11)$ & $14(11-17)$ & $<0.001$ & yes \\
\hline \multicolumn{5}{|l|}{$\begin{array}{l}\text { Phospholipids in } \\
\text { subclasses }\end{array}$} \\
\hline VLDL (mg/dL) & $22(15-41)$ & $15(11-28)$ & 0.039 & no \\
\hline IDL (mg/dL) & $5(3-11)$ & $4(2-6)$ & 0.133 & no \\
\hline $\mathrm{LDL}(\mathrm{mg} / \mathrm{dL})$ & $40(28-55)$ & $51(34-62)$ & 0.095 & no \\
\hline HDL (mg/dL) & $36(18-51)$ & $62(49-73)$ & $<0.001$ & yes \\
\hline \multicolumn{5}{|l|}{$\begin{array}{c}\text { Apolipoproteins in } \\
\text { subclasses }\end{array}$} \\
\hline ApoA1 in HDL (mg/dL) & $67(46-98)$ & $119(98-138)$ & $<0.001$ & yes \\
\hline ApoA2 in HDL (mg/dL) & $20(17-25)$ & $25(21-27)$ & 0.006 & no \\
\hline ApoB in VLDL (mg/dL) & $18(11-27)$ & $8(7-13)$ & $<0.001$ & yes \\
\hline
\end{tabular}


Table 2. Cont.

\begin{tabular}{lcccc}
\hline Variables & Sepsis Patients $(\mathbf{N}=\mathbf{5 2})$ & Controls $(\mathbf{N}=\mathbf{2 5})$ & $p$-Value & Below Sidak-Threshold * \\
\hline ApoB in IDL $(\mathrm{mg} / \mathrm{dL})$ & $9(4-17)$ & $5(3-8)$ & 0.002 & no \\
\hline ApoB in LDL $(\mathrm{mg} / \mathrm{dL})$ & $51(41-67)$ & $57(40-69)$ & 0.640 & no \\
\hline * -Value corrections for multiple testing were performed with the Sidak-method (lower values than the \\
threshold are significant)-Sidak-Treshold at 0.00044984. Data are reported as medians (25th-75th percentile). \\
Abbreviations: ICU = intensive care unit; VLDL = very low-density lipoprotein; LDL = low-density lipoprotein; \\
IDL = intermediate-density lipoprotein; HDL = high-density lipoprotein; ApoA1 = apolipoprotein A-I; ApoA2 = apolipoprotein \\
A-II; ApoB100 = apolipoprotein B-100.
\end{tabular}

\subsection{Untargeted Metabolomic Assessment of Metabolites in the Sepsis Cohort}

In a second step, we extracted metabolites from serum samples to further investigate differences between sepsis and septic shock patients, as well as septic survivors and nonsurvivors. This approach allows for an untargeted metabolomic analysis to assess for differences of metabolic phenotypes between groups, which is more challenging in a complex blood sample matrix containing high concentrations of lipoproteins, masking metabolite signals. Moreover, a higher number of metabolites can be identified in this approach. In PCA analysis, we found differences between the four groups of survivors and non-survivors each for the shock and no-shock group with a PC1 of 55\% and PC2 of $14.3 \%$ (Figure 2A). Pair-wise O-PLS-DA comparisons showed acceptable clustering for survivors and non-survivors in the shock group and for the comparison of no-shock and shock in the survivor group with cross-validation scores $Q^{2}$ of 0.346 and 0.438 , respectively (each $p<0.01$, Figure 2B).

Blood serum levels of the branched-chain amino acids (BCAA) valine (33.0 vs. 55.0; $p=0.002)$, leucine (53.4 vs. $70.8 ; p=0.005)$ and isoleucine (15.2 vs. $18.1 ; p=0.012$ ) were significantly lower in ICU non-survivors compared to ICU survivors (Table 3, Figure 2C). In the shock group, BCAA and 3-hydroxybutyrate (3-HB) were lower in non-survivors compared to survivors, while levels of other unassignable metabolites were higher in non-survivors (Figure 2D). Similarly, valine ( 43.3 vs. $64.3 ; p=0.005$ ), leucine ( 57.0 vs. 73.0 ; $p=0.034)$, and isoleucine (15.2 vs. 17.9; $p=0.048)$ levels were lower in shock patients compared to no-shock patients. Lactate, a marker in the definition of septic shock, served as an internal control, and levels were, as expected, higher in septic shock patients compared to those without shock (Figure 2E). In a sub-analysis, after the exclusion of patients who received propofol or renal replacement therapy (RRT), the results for the corresponding metabolite analyses remained similar.

\subsection{Correlations between Metabolites and Other Parameters in the Sepsis Cohort}

Higher SOFA scores as a marker for more severe organ dysfunction significantly inversely correlated with valine $(\mathrm{r}=-0.338, p=0.01)$, isoleucine $(\mathrm{r}=-0.284, p=0.04)$, but not leucine $(-0.220, p=0.114$; Table 4$)$. Valine correlated with other BCAA and inversely correlated with PCT $(r=-0.401, p=0.003)$ and CRP $(r=-0.271, p=0.05)$. Both leucine and isoleucine inversely correlated with PCT, but not CRP. 
A

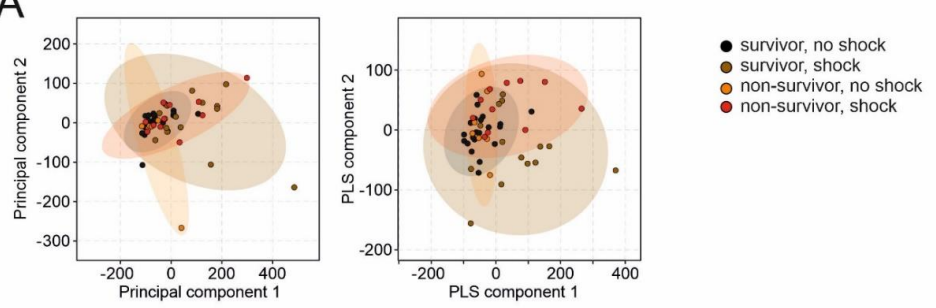

B

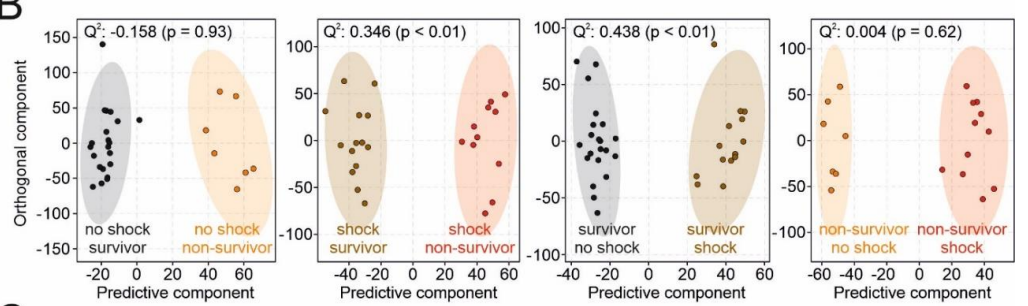

C
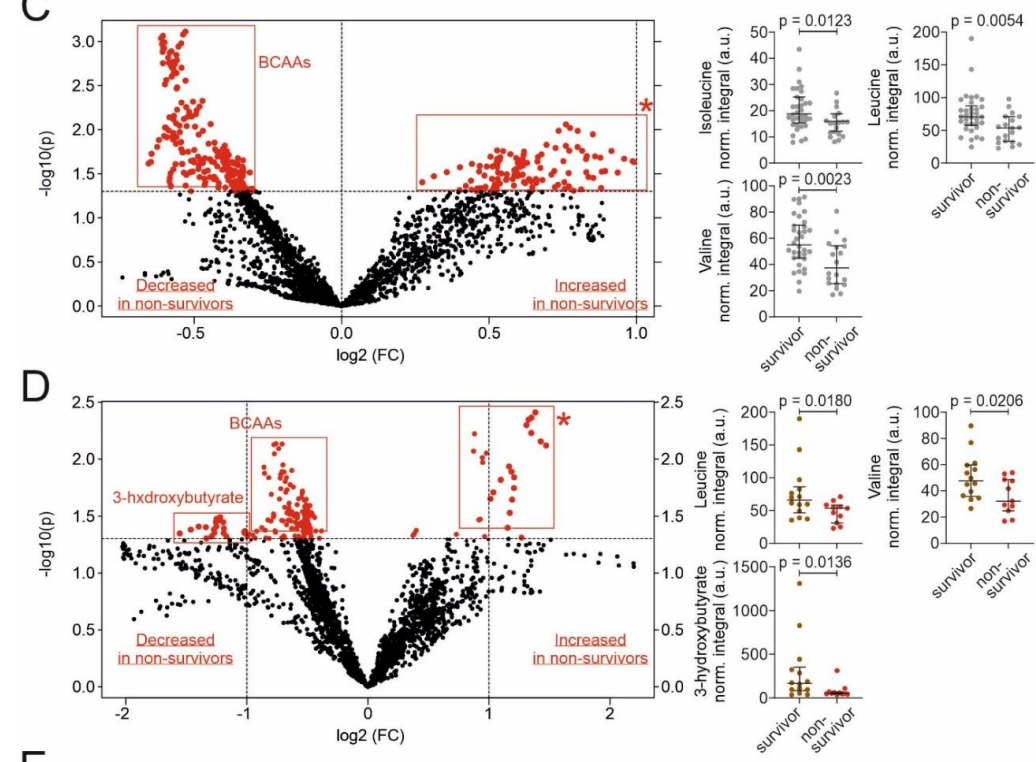

E



Figure 2. Untargeted metabolomic assessment of metabolites in sepsis. (A) Principal component analysis (PCA; left panel) and partial least squares discriminant analysis (PLS-DA; right panel) for the differentiation between the four groups of survivors and non-survivors, each for the shock and no-shock group. PCA: principal component (PC) 1 of $55 \%$ and PC2 of 14.3\%. PLS-DA: Component 1 of 50.4\%, Component 2 of $14.6 \%$. (B) Pair-wise orthogonal partial least squares discriminant analysis (O-PLS-DA) showing acceptable clustering for survivors and non-survivor in the shock group (middle-left panel; $\mathrm{Q}^{2}=0.346, p<0.01$ ), as well as survivors of the no-shock and shock group (middle-right panel; $\mathrm{Q}^{2}=0.438, p<0.01$ ). No significant clustering was found for survivors and non-survivor in the no-shock group (left panel; $p=0.93$ ) and for non-survivors of the no-shock and shock group (right panel; $p=0.62$ ). (C) Differences in metabolites of all sepsis patients between survivors and non-survivors (volcano plot). The horizontal dashed line marks the level of significance at 0.05 . The boxplots represent the most pronounced and most significant different metabolites. (D) Differences in metabolites of septic shock patients between survivors and non-survivors (volcano plot). The horizontal dashed line marks the level of significance at 0.05 . The boxplots represent the most pronounced and most significant different metabolites. (E) Comparison between no-shock and septic shock patients. Boxplots of the most pronounced and most significant different metabolites. Abbreviations: BCAA = branched-chain amino acids (consisting of valine, leucine, and isoleucine). a.u. = arbitrary unit. * = unassigned metabolites. 
Table 3. Assigned and most prominent metabolic changes with sepsis and septic shock determined by untargeted ${ }^{1} \mathrm{H}$ NMR spectroscopy.

\begin{tabular}{|c|c|c|c|c|c|c|c|}
\hline Variable & $\begin{array}{l}\text { Sepsis Cohort } \\
\quad N=53\end{array}$ & $\begin{array}{l}\text { Shock } \\
N=26\end{array}$ & $\begin{array}{c}\text { No-Shock } \\
\mathbf{N}=27\end{array}$ & $p$-Value & $\begin{array}{c}\text { ICU-Survivors } \\
\mathbf{N}=34\end{array}$ & $\begin{array}{c}\text { Non-Survivors } \\
\mathbf{N}=19\end{array}$ & $p$-Value \\
\hline \multicolumn{8}{|l|}{ Metabolomic results } \\
\hline Valine & 49.7 (33.3-65.6) & $43.3(29.0-53.7)$ & $64.3(47.7-72.3)$ & 0.005 & $55.0(44.8-70.2)$ & $33.0(24.9-53.9)$ & 0.002 \\
\hline Leucine & $65.3(44.1-81.3)$ & $57.0(38.4-71.0)$ & $73.0(54.3-86.3)$ & 0.034 & $70.8(57.8-87.5)$ & $53.4(32.0-71.0)$ & 0.005 \\
\hline Isoleucine & $17.0(13.7-22.4)$ & $15.2(10.9-21.6)$ & $17.9(16.1-24.4)$ & 0.048 & $18.1(14.6-24.6)$ & $15.2(11.1-17.9)$ & 0.012 \\
\hline Acetate * & $35.6(27.2-44.3)$ & $35.8(30.4-48.4)]$ & $30.9(24.5-44.1)$ & 0.292 & $34.8(29.5-46.4)$ & $35.6(24.1-48.6)$ & 1.000 \\
\hline Phenylalanine & $33.3(23.7-47.4)$ & $35.6(25.8-48.0)$ & $27.9(21.9-47.3)$ & 0.188 & $30.0(23.6-47.4)$ & $36.0(24.2-48.2)$ & 0.458 \\
\hline Tyrosine & $8.1(6.3-11.3)$ & $8.2(6.2-12.6)$ & $8.1(6.3-11.3)$ & 0.715 & $8.7(7.0-11.5)$ & $7.0(5.6-9.3)$ & 0.156 \\
\hline Lactate & 587 (383-914) & $815(586-1394)$ & $409(301-601)$ & $<0.0001$ & $566(392-995)$ & $587(347-890)$ & 0.970 \\
\hline Citrate & $22.8(19.1-28.3)$ & $24.3(18.6-29.7)$ & $22.5(19.2-25.5)$ & 0.466 & $22.7(19.1-28.7)$ & $23.5(19.1-27.3)$ & 0.970 \\
\hline
\end{tabular}


Table 4. Heatmap of correlations of selected variables and metabolites in the sepsis cohort.

\begin{tabular}{|c|c|c|c|c|c|c|c|c|c|c|c|c|c|c|}
\hline Variables & Age & Valine & Leucine & Isoleucine & Acetate & 3-HB & Phenyl-alanine & Tyrosine & Citrate & Lactate & BMI & CRP & PCT & IL -6 \\
\hline SOFA & -0.18 & -0.338 * & -0.220 & $-0.284^{*}$ & -0.226 & -0.219 & 0.078 & -0.021 & 0.004 & 0.198 & 0.136 & 0.097 & $0.378^{* *}$ & 0.344 * \\
\hline Age & & -0.050 & -0.042 & 0.078 & 0.011 & 0.184 & 0.037 & -0.097 & 0.001 & -0.012 & 0.041 & 0.224 & -0.141 & -0.069 \\
\hline Valine & & & $0.860 * *$ & $0.833^{* *}$ & 0.321 * & 0.145 & $-0.319 *$ & $0.566^{* *}$ & -0.004 & -0.009 & 0.084 & $\begin{array}{c}-0.271 \\
*\end{array}$ & $-0.401^{* *}$ & $-0.394^{* *}$ \\
\hline Leucine & & & & $0.798^{* *}$ & $0.310^{*}$ & 0.298 * & $-0.304^{*}$ & $0.448^{* *}$ & 0.059 & -0.002 & -0.052 & -0.223 & $-0.396^{* *}$ & $-0.375^{* *}$ \\
\hline Isoleucine & & & & & 0.215 & 0.303 * & -0.271 * & $0.564^{* *}$ & 0.108 & 0.021 & 0.043 & -0.208 & $-0.408^{* *}$ & -0.270 \\
\hline Acetate & & & & & & 0.194 & -0.109 & $0.287^{*}$ & -0.124 & 0.320 * & -0.010 & -0.428 & -0.219 & -0.292 * \\
\hline $3-\mathrm{HB}$ & & & & & & & -0.053 & -0.017 & -0.082 & 0.045 & 0.039 & 0.006 & $-0.276^{*}$ & -0.130 \\
\hline Phenylalani & & & & & & & & 0.004 & 0.206 & 0.041 & 0.218 & 0.035 & 0.267 & 0.263 \\
\hline Tyrosine & & & & & & & & & 0.006 & $0.436^{* *}$ & 0.160 & $\begin{array}{c}-0.476 \\
* *\end{array}$ & -0.125 & -0.024 \\
\hline Citrate & & & & & & & & & & 0.090 & 0.048 & 0.055 & -0.112 & 0.096 \\
\hline Lactate & & & & & & & & & & & 0.149 & -0.114 & 0.120 & 0.268 \\
\hline BMI & & & & & & & & & & & & -0.040 & 0.036 & 0.015 \\
\hline CRP & & & & & & & & & & & & & 0.255 & $0.375^{* *}$ \\
\hline
\end{tabular}

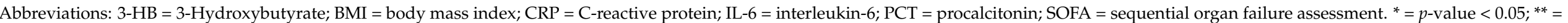
$p$-value $<0.01$. Dark red : very strong correlation $(+/->0.65)$; Red : strong correlation $(+/-0.55-0.65)$; Orange : moderate correlation $(+/-0.45-0.55) ;$ Light blue : weak correlation $(+/-0.35-0.45)$; very weak correlation $(+/-0.25-0.35)$; Dark blue : no correlation $(+/-<0.25)$. 


\subsection{Univariable and Multivariable Regression Analyses}

Univariable logistic regression was performed both for 28-day and ICU-mortality. In the sepsis cohort, for ICU-mortality only SOFA score and BCAA serum levels were significantly predictive for the outcome (Table 5). A lower SOFA score as wells as higher valine levels (odds ratio (OR) per doubling $=0.19,95 \%$ CI: $0.06-0.58, p=0.004$ ), higher leucine levels (OR per doubling $=0.22,95 \%$ CI: $0.07-0.66, p=0.007$ ), and higher isoleucine levels (OR per doubling $=0.23,95 \%$ CI: $0.07-0.81, p=0.023$ ) were associated with reduced ICU-mortality. Similarly, several variables partly predicted 28-day mortality in our sepsis cohort. Older age, higher CRP, lower valine, leucine, and isoleucine serum levels were associated with increased 28-day mortality. In multivariable analyses, valine levels remained predictive for ICU and 28-day mortality (Table 6; Supplementary Tables S4 and S5). The 28-day Kaplan-Meier survival estimates for patients below the 25th percentile compared to those above this cutoff were $14 \%$ vs. $66 \%$ for valine (log-rank $p=0.0001), 21 \%$ vs. $64 \%$ for leucine ( $\log \operatorname{rank} p=0.003$ ) and $29 \%$ vs. $61 \%$ for isoleucine (log-rank $p=0.003$; Figure 3 ).

Table 5. Univariable logistic regression models in sepsis patients for prediction of 28-day and ICU mortality.

\begin{tabular}{|c|c|c|c|c|c|c|}
\hline \multirow{2}{*}{\begin{tabular}{|l}
$\begin{array}{l}\text { Outcome } \\
\text { Variable }\end{array}$ \\
Variable
\end{tabular}} & \multicolumn{3}{|c|}{ 28-Day Mortality } & \multicolumn{3}{|c|}{ ICU Mortality } \\
\hline & Odds Ratio & $\begin{array}{l}\text { 95\% Confidence } \\
\text { Interval }\end{array}$ & $p$ & Odds Ratio & $\begin{array}{l}95 \% \text { Confidence } \\
\text { Interval }\end{array}$ & $p$ \\
\hline \multicolumn{7}{|l|}{ Demographics } \\
\hline $\begin{array}{c}\text { Age (per } 5 \\
\text { years increase) }\end{array}$ & 1.23 & $1.02-1.50$ & 0.033 & 1.06 & $0.89-1.27$ & 0.511 \\
\hline Female sex & 2.71 & $0.87-8.42$ & 0.085 & 1.65 & $0.53-5.17$ & 0.390 \\
\hline $\begin{array}{c}\text { Anti-diabetic } \\
\text { therapy }\end{array}$ & 0.75 & $0.20-2.75$ & 0.665 & 0.87 & $0.22-3.37$ & 0.836 \\
\hline Type 2 diabetes & 0.45 & $0.13-1.57$ & 0.210 & 0.56 & $0.15-2.08$ & 0.384 \\
\hline Liver disease & 2.35 & $0.20-27.6$ & 0.497 & 3.88 & $0.33-45.93$ & 0.282 \\
\hline \multicolumn{7}{|l|}{$\begin{array}{l}\text { Laboratory } \\
\text { parameters }\end{array}$} \\
\hline $\begin{array}{c}\text { White blood } \\
\text { count } \\
\text { (per } 1 \mathrm{G} / \mathrm{L} \\
\text { increase) }\end{array}$ & 1.02 & 0.98-1.07 & 0.357 & 1.00 & $0.96-1.05$ & 0.960 \\
\hline $\begin{array}{l}\text { Hemoglobin } \\
\text { (per } 1 \mathrm{~g} / \mathrm{dL} \\
\text { increase) }\end{array}$ & 0.94 & $0.77-1.15$ & 0.574 & 1.03 & $0.84-1.25$ & 0.801 \\
\hline $\begin{array}{c}\text { Platelets (per } \\
100 \mathrm{G} / \mathrm{L} \\
\text { increase) }\end{array}$ & 1.11 & $0.71-1.75$ & 0.640 & 1.14 & $0.71-1.81$ & 0.593 \\
\hline $\begin{array}{c}\text { C-reactive } \\
\text { protein } \\
\text { (per } 100 \mathrm{mg} / \mathrm{L} \\
\text { increase) }\end{array}$ & 1.72 & $1.07-2.77$ & 0.025 & 1.40 & $0.90-2.18$ & 0.136 \\
\hline $\begin{array}{l}\text { Procalcitonin } \\
\text { (per } 10 \mathrm{ng} / \mathrm{mL} \\
\text { increase) }\end{array}$ & 1.03 & $0.97-1.09$ & 0.339 & 1.04 & $0.98-1.11$ & 0.170 \\
\hline $\begin{array}{c}\text { Serum } \\
\text { creatinine } \\
\text { (per } 1 \mathrm{mg} / \mathrm{dL} \\
\text { increase) }\end{array}$ & 1.01 & 0.85-1.19 & 0.905 & 1.04 & $0.88-1.23$ & 0.653 \\
\hline $\begin{array}{l}\text { Serum bilirubin } \\
\text { (per } 1 \mathrm{mg} / \mathrm{dL} \\
\text { increase) }\end{array}$ & 0.89 & $0.74-1.08$ & 0.245 & 0.94 & $0.80-1.11$ & 0.484 \\
\hline
\end{tabular}


Table 5. Cont.

\begin{tabular}{|c|c|c|c|c|c|c|}
\hline $\begin{array}{l}\text { Outcome } \\
\text { Variable }\end{array}$ & \multicolumn{3}{|c|}{ 28-Day Mortality } & \multicolumn{3}{|c|}{ ICU Mortality } \\
\hline Variable & Odds Ratio & $\begin{array}{c}\text { 95\% Confidence } \\
\text { Interval }\end{array}$ & $p$ & Odds Ratio & $\begin{array}{c}95 \% \text { Confidence } \\
\text { Interval }\end{array}$ & $p$ \\
\hline \multicolumn{7}{|l|}{ Metabolites } \\
\hline $\begin{array}{l}\text { Valine (per } \\
\text { doubling) }\end{array}$ & 0.18 & $0.06-0.56$ & 0.003 & 0.19 & $0.06-0.58$ & 0.004 \\
\hline $\begin{array}{l}\text { Leucine (per } \\
\text { doubling) }\end{array}$ & 0.19 & $0.06-0.59$ & 0.004 & 0.22 & $0.07-0.66$ & 0.007 \\
\hline $\begin{array}{l}\text { Isoleucine (per } \\
\text { doubling) }\end{array}$ & 0.29 & $0.09-0.93$ & 0.038 & 0.23 & $0.07-0.81$ & 0.023 \\
\hline $\begin{array}{l}\text { Acetate (per } \\
\text { doubling) }\end{array}$ & 1.26 & $0.57-2.80$ & 0.572 & 1.24 & $0.54-2.85$ & 0.609 \\
\hline $\begin{array}{l}\text { 3- } \\
\text { Hydroxybutyrate } \\
\text { (per doubling) }\end{array}$ & 0.91 & $0.61-1.38$ & 0.668 & 0.79 & $0.50-1.26$ & 0.326 \\
\hline $\begin{array}{l}\text { Phenylalanine } \\
\text { (per doubling) }\end{array}$ & 1.77 & $0.75-4.19$ & 0.194 & 1.23 & $0.53-2.88$ & 0.631 \\
\hline $\begin{array}{l}\text { Tyrosine (per } \\
\text { doubling) }\end{array}$ & 0.83 & $0.35-1.95$ & 0.665 & 0.82 & $0.33-2.04$ & 0.675 \\
\hline $\begin{array}{l}\text { Lactate (per } \\
\text { doubling) }\end{array}$ & 1.15 & $0.65-2.04$ & 0.632 & 1.00 & $0.55-1.81$ & 0.996 \\
\hline $\begin{array}{l}\text { Citrate (per } \\
\text { doubling) }\end{array}$ & 1.41 & $0.44-4.54$ & 0.563 & 0.90 & $0.27-3.03$ & 0.865 \\
\hline \multicolumn{7}{|l|}{$\begin{array}{l}\text { Sepsis severity } \\
\text { score }\end{array}$} \\
\hline $\begin{array}{l}\text { SOFA score (per } \\
1 \text { point } \\
\text { increase) }\end{array}$ & 1.13 & $0.97-1.31$ & 0.113 & 1.36 & $1.12-1.65$ & 0.002 \\
\hline \multicolumn{7}{|c|}{$\begin{array}{l}\text { Abbreviations: SOFA = sequential organ failure assessment. The odds ratio per doubling of the predictor variable was obtained by a } \\
\log _{2}(x+1) \text { transformation of the variable. Note that metabolites are measured in normalized signal intensity units. }\end{array}$} \\
\hline \multicolumn{2}{|c|}{$\begin{array}{l}\text { Multivariable Model 1: } \\
\text { 28-Day Mortality }\end{array}$} & Odds Ratio & \multicolumn{2}{|c|}{ 95\% Confidence Interval } & \multicolumn{2}{|c|}{$p$} \\
\hline \multicolumn{2}{|c|}{ Age (per 5 year increase) } & 1.25 & \multicolumn{2}{|r|}{$1.00-1.56$} & \multicolumn{2}{|c|}{0.048} \\
\hline \multicolumn{2}{|c|}{$\begin{array}{c}\text { C-reactive protein (per } 100 \\
\mathrm{mg} / \mathrm{L} \text { increase) }\end{array}$} & 1.37 & \multicolumn{2}{|r|}{$0.80-2.35$} & \multicolumn{2}{|c|}{0.257} \\
\hline \multicolumn{2}{|c|}{ Valine (per doubling) } & 0.19 & \multicolumn{2}{|r|}{$0.05-0.66$} & \multicolumn{2}{|c|}{0.009} \\
\hline \multicolumn{2}{|c|}{$\begin{array}{l}\text { Multivariable Model 2: ICU } \\
\text { Mortality }\end{array}$} & Odds Ratio & \multicolumn{2}{|c|}{ 95\% Confidence Interval } & \multicolumn{2}{|c|}{$p$} \\
\hline \multicolumn{2}{|c|}{$\begin{array}{c}\text { SOFA score (per } 1 \text { point } \\
\text { increase) }\end{array}$} & 1.29 & \multicolumn{2}{|r|}{$1.06-1.57$} & \multicolumn{2}{|c|}{0.012} \\
\hline \multicolumn{2}{|c|}{ Valine (per doubling) } & 0.26 & & $0.08-0.85$ & \multicolumn{2}{|c|}{0.026} \\
\hline
\end{tabular}

Abbreviations: ICU = intensive care unit. SOFA = sequential organ failure assessment. The odds ratio per doubling of the predictor variable were obtained by a $\log _{2}(x+1)$ transformation of the variable. 
(A)

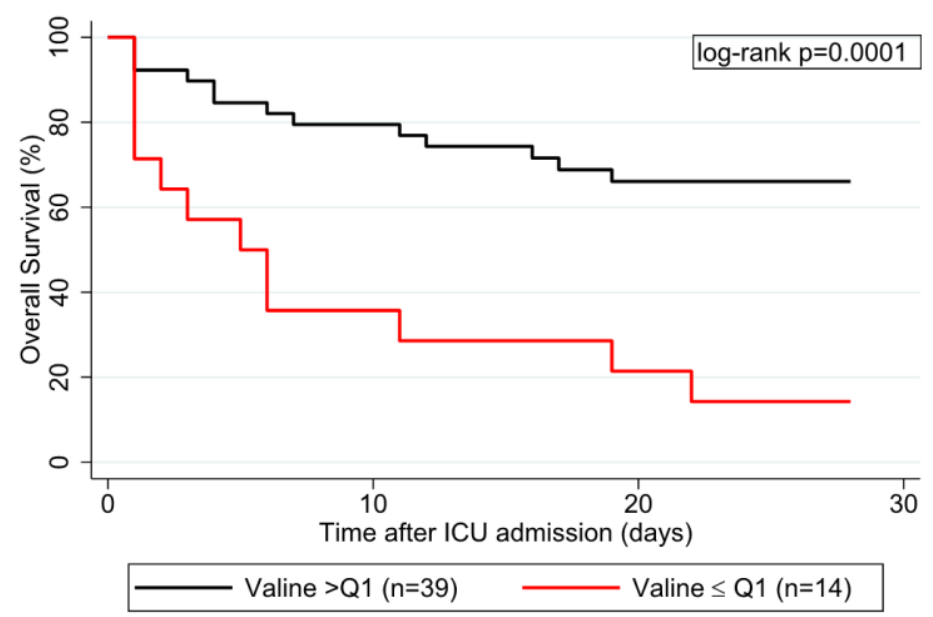

(B)

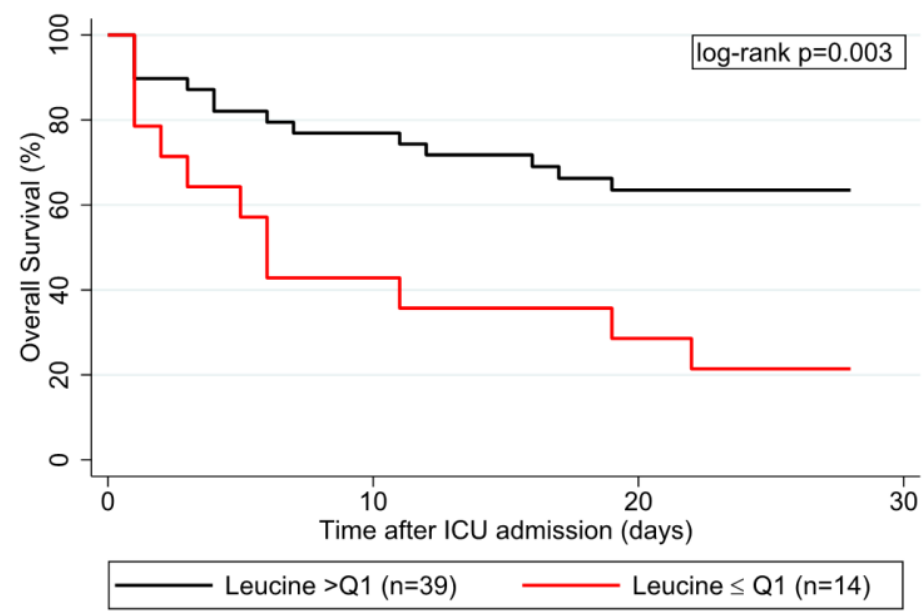

(C)

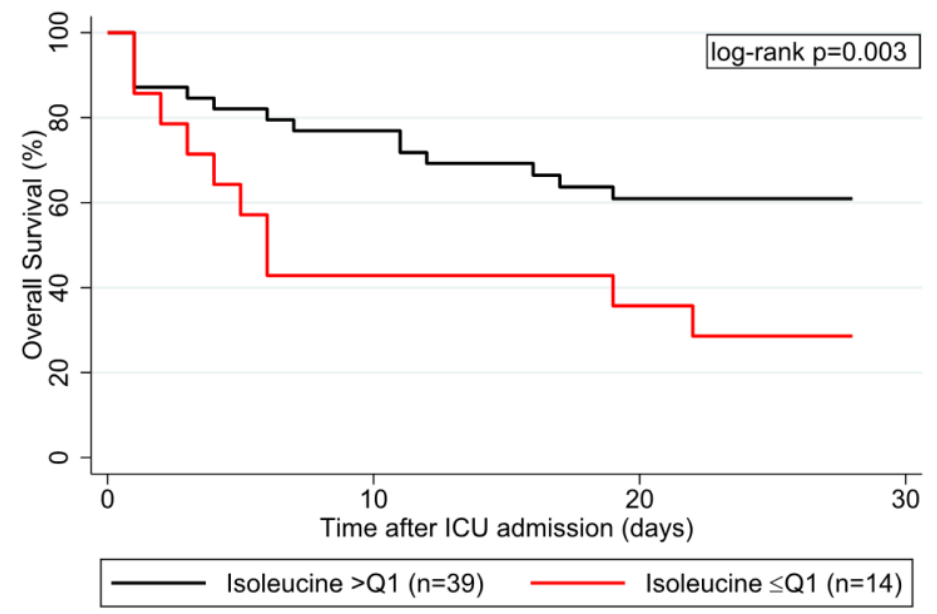

Figure 3. Kaplan-Meier estimates for valine (A), leucine (B), and isoleucine (C). The Q1, i.e., the 25th percentile, corresponds to a cutoff value for valine, leucine, and isoleucine of 33.3, 44.1, and 13.7 NSI units, respectively. The 28-day Kaplan-Meier survival estimates for patients below or above the respective cutoff were $14 \%$ vs. $66 \%$ for valine, $21 \%$ vs. $64 \%$ for leucine, and $29 \%$ vs. $61 \%$ for isoleucine. Abbreviations: $\mathrm{Q} 1=25$ th percentile of the respective branched-chain amino acid, ICU $=$ intensive care unit. 


\subsection{Area under the Receiver Operating Characteristics (AUROC) Curve}

The SOFA score and all three BCAA were strong discriminators regarding ICUmortality (Table 7, Figure 4A). The BCAAs were furthermore strong discriminators for 28-day mortality, while the SOFA score was a weak discriminator for 28-day mortality in our cohort. In an exploratory analysis, a SOFA score to BCAA ratio was an even stronger discriminator of ICU-mortality (Figure 4B).

Table 7. ROC curves for 28-day and ICU mortality.

\begin{tabular}{ccccc}
\hline Outcome & \multicolumn{2}{c}{ ICU Mortality } & \multicolumn{2}{c}{ 28-day Mortality } \\
\hline Variables & AUROC & 95\% Confidence Interval & AUROC & 95\% Confidence Interval \\
\hline SOFA score & 0.78 & $0.65-0.91$ & 0.62 & $0.46-0.78$ \\
\hline Valine & 0.75 & $0.62-0.89$ & 0.75 & $0.62-0.89$ \\
\hline Leucine & 0.73 & $0.59-0.88$ & 0.75 & $0.62-0.88$ \\
\hline Isoleucine & 0.71 & $0.57-0.85$ & 0.69 & $0.54-0.83$ \\
\hline SOFA & 0.85 & $0.73-0.96$ & 0.74 & $0.60-0.88$ \\
\hline
\end{tabular}

Abbreviations: AUROC $=$ area under the receiver operating curve. $I C U=$ intensive care unit. SOFA $=$ sequential organ failure assessment. $\mathrm{BCAA}=$ branched-chain amino acids (valine + leucine + isoleucine)

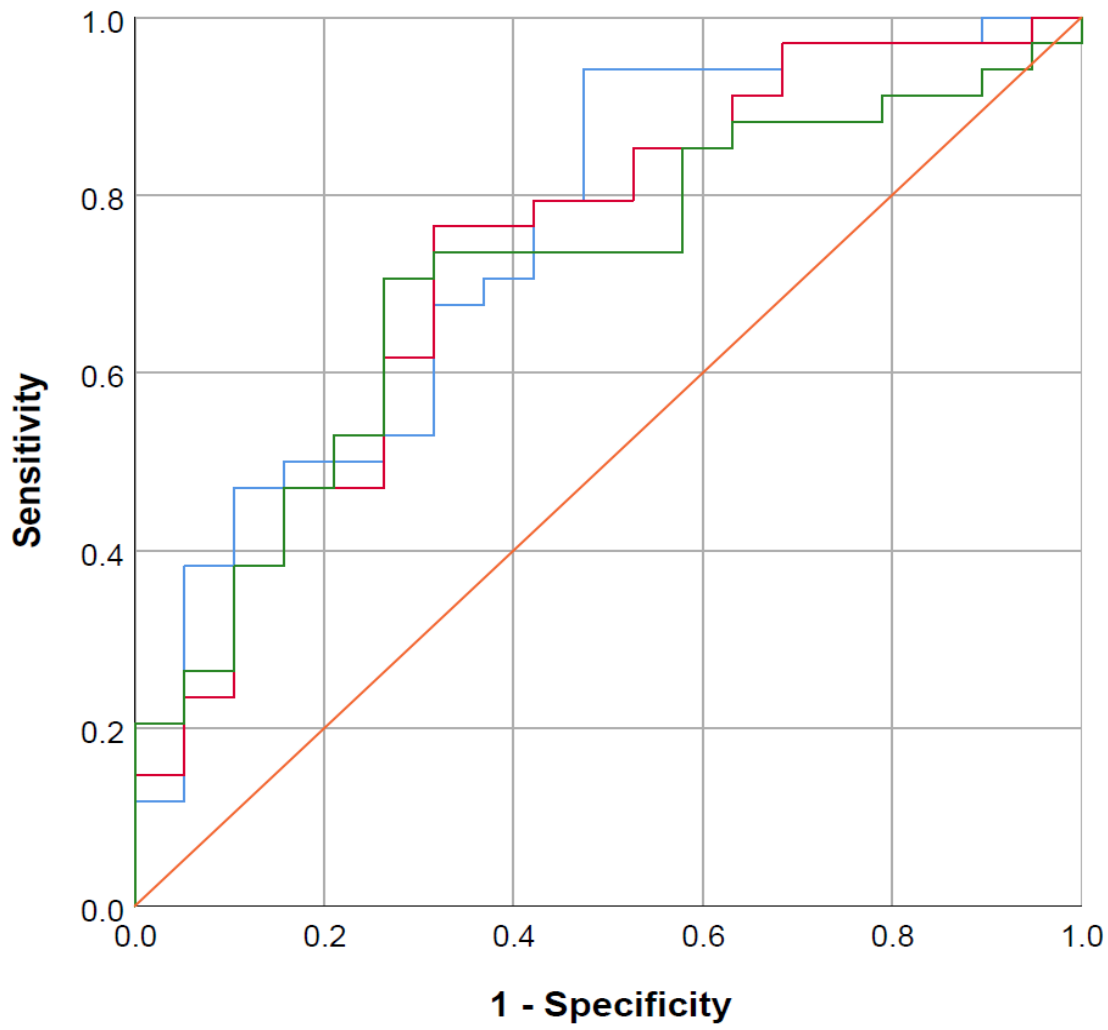

\section{ROC curves}

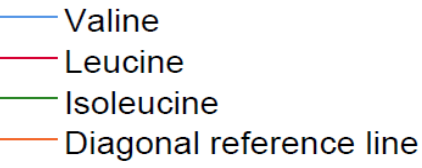

(A)

Figure 4. Cont. 


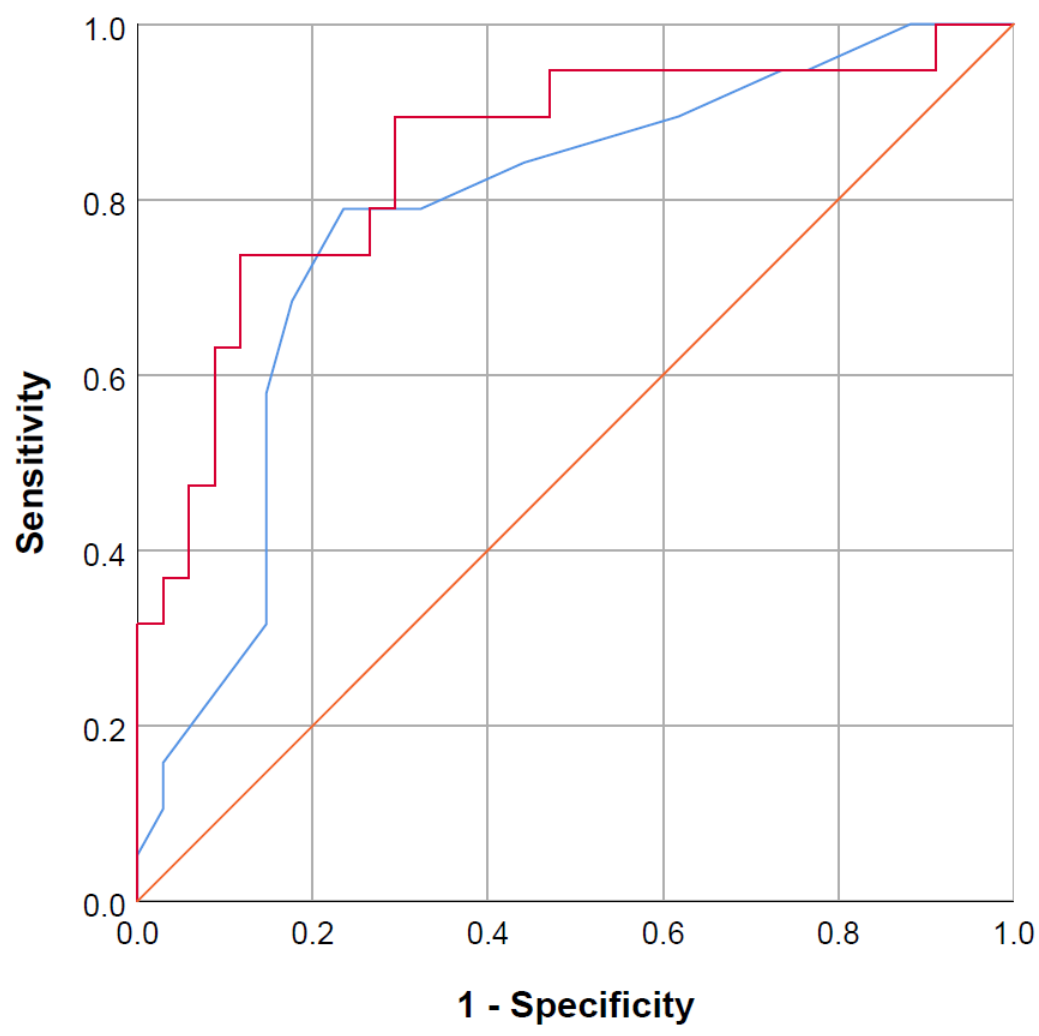

ROC curves

SOFA score

- SOFA score to BCAA ratio

- Diagonal reference line

(B)

Figure 4. Area under the receiver operating characteristics (AUROC) of branched-chain amino acids for ICU-mortality. (A): AUROC for valine (blue line, 0.75 [0.62-0.89]), leucine (red line, $0.73(0.59-0.88)$ ) and isoleucine (green line, 0.71 (0.57-0.85)). (B): AUROC for the sequential organ failure assessment (SOFA) score (blue line, $0.78(0.65-0.91)$ ) and the SOFA-score to branched-chain amino acid ratio (red line, $0.85(0.73-0.96)$ ). The orange line is the diagonal reference line (50\% chance).

\subsection{Longitudinal Data during the ICU Stay for Sepsis Patients}

Samples on day three and day seven after ICU admission were available for 30 and 16 patients, respectively. To assess for potential bias, we compared patients in whom samples from day three or seven were available to those without samples on these days (already discharged or deceased) but found that the groups were not statistically significantly different (Supplementary Table S6). In a linear mixed model for the BCAAs we found that ICU survivors compared to non-survivors had similar values in our cohort over time. The ICU non-survivor subgroup got enriched over time with patients having higher valine levels, suggesting that higher valine levels may be associated with prolonged time to death in patients succumbing on ICU (Figure 5). 

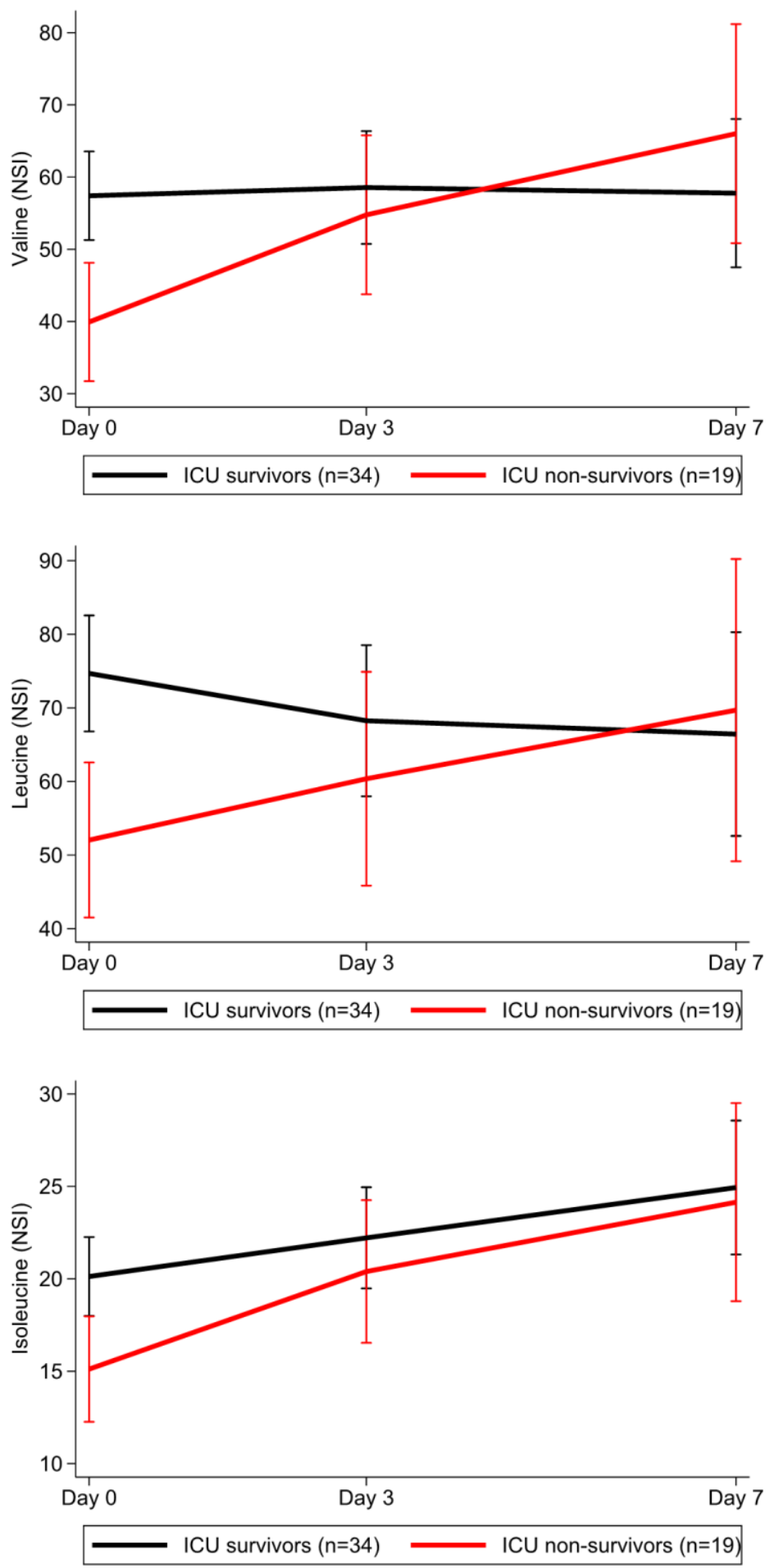

Figure 5. Longitudinal data of branched-chain amino acid levels. Mean levels of branched-chain amino acids (top panel: valine; middle panel: leucine; bottom panel: isoleucine) over time in sepsis survivors (black line) and sepsis non-survivors (red line) from day 0 to day 3 and 7 after admission. Survivors compared to non-survivors had significantly higher levels of branched-chain amino acids on day 0 . Note that in the non-survivor cohort, lower branched-chain amino acids were associated with decreased time until death. 


\section{Discussion}

In our study, we assessed the metabolomic profiles of patients with sepsis and septic shock who were admitted to the ICU and ICU patients without sepsis or bacteremia. Sepsis and septic shock are common in intensive care medicine and have high mortality rates $[15,16]$. The diagnosis is based on clinical criteria and includes a suspected infection by the treating physician and the presence of new organ dysfunctions [3]. The SOFA score is used to semi-quantitatively assess the severity of organ dysfunction and serves as a prognostic outcome marker. Several biomarkers in sepsis have been investigated without convincing results regarding the prognostic and diagnostic potential [17]. Established therapies in sepsis include early source control and early anti-infective therapy, while specific anti-inflammatory or pro-inflammatory therapies have failed [18]. Better prognostic biomarkers and therapeutic targets are urgently needed. In this study, we used ${ }^{1} \mathrm{H}$ NMR spectroscopy to perform both a targeted analysis for lipoproteins as well as an untargeted approach to investigate a broad spectrum of metabolites simultaneously. Therefore, metabolites that were previously unknown to play a role may be detected. Furthermore, metabolomics, in contrast to genomics or proteomics, reflects the metabolites present at a given timepoint that are responsible for direct or indirect effects in the human body. Metabolites represent key molecules reflecting dietary and disease related pathway dysfunctions and may therefore provide an effective prognostic value and possible target for therapeutic intervention.

We were able to show in our study that valine, an essential amino acid, which must be consumed by protein-rich dietary products, was significantly reduced in non-survivors as compared to sepsis survivors. In addition, non-survivors compared to ICU survivors had lower levels of leucine and isoleucine. These three metabolites comprise the branchedchain amino acids (BCAA) group and are needed for protein synthesis and muscle strength BCAA moreover inhibit proteolysis, improve immune function, and activate mTOR pathways [19-21]. Previously, higher levels of valine were found in diabetic humans with insulin resistance $[22,23]$. Insulin resistance leads to decreased glucose utilization and inhibited lipolysis, resulting in muscle protein degradation [24]. In our study, levels of valine were lower with increasing sepsis severity, despite insulin resistance and protein degradation, with skeletal muscle wasting being a common feature of septic patients $[25,26]$. Therefore, the BCAA catabolism must exceed the increased levels caused by insulin resistance and protein breakdown [19]. Another possible explanation for the low valine levels in sepsis may be an increased branched-chain alpha-keto acid dehydrogenase (BCKD) activity leading to increased oxidation of BCAA [27]. Liver failure or dysfunction is unlikely to play a role, as BCAA metabolism occurs primarily in extrahepatic tissues $[28,29]$. In our study, BCAA levels were furthermore not correlated with liver parameters such as bilirubin. As we cannot provide pre-admission laboratory values, it may be possible that people with low BCAA levels have a higher risk of developing sepsis and, in addition, have a worse prognosis when ICU admission is necessary. However, animal studies suggest that BCAA levels significantly decrease during LPS stimulation [30,31] and other small studies in humans also found decreased levels in sepsis patients [32,33]. Therefore, a decrease during sepsis instead of pre-existing low BCAA levels is more likely to account for the majority of BCAA scarcity, especially as the SOFA score as a marker for organ dysfunction is inversely correlated with valine and isoleucine.

In general, our data are mostly consistent with other studies showing reduced BCAA levels in sepsis, including a small study by Freund et al. that demonstrated higher levels of BCAA in sepsis survivors compared to non-survivors [24]. In addition, Puskarich et al. have found BCAA to be predictors for shock resolution in sepsis [34]. In another study comparing septic shock patients with and without encephalopathy, those with encephalopathy had higher levels of ammonia, phenylalanine, tryptophan, GABA, urea, and lower levels of isoleucine, cysteine, glutamine, and arginine [35]. In a more recent study by Liu et al., levels of ketone bodies, phenylalanine, glutamate, lactate, and others were significantly different between septic shock survivors and non-survivors, but no results on BCAA were pro- 
vided [36]. Mickiewicz et al. investigated septic shock patients and found elevated levels of phenylalanine, lactate, 3-HB, as well as reduced levels of BCAAs and other metabolites compared to ICU controls [37,38]. In another study, valine, beta-hydroxybutyrate, citrate, and other metabolites in trauma patients were found to be predictors for later sepsis development [39]. In our study, we also showed reduced levels of BCAA in patients suffering from sepsis with lower levels in patients suffering from more severe disease. Furthermore, in the shock group, BCAA and 3-HB were lower in non-survivors compared to survivors. We did, however, only investigate sepsis patients for those metabolites, which may explain the differences in our study compared to the study by Mickiewicz and colleagues. On the other hand, lactate was one of the significantly different metabolites between shock and no-shock sepsis patients, which is based on the current diagnostic criteria for septic shock, and therefore provided an internal validation of our data. Su et al. found lower values of lactitol dehydrate and S-phenyl-D-cysteine and increased values of S-(3-methylbutanoyl)dihydrolipoamide-E and N-nonanoyl-glycine in sepsis compared to SIRS patients [40]. S-(3-methylbutanoyl)-dihydrolipoamide is relevant for the degradation of BCAAs, which may explain the increased levels in sepsis patients [6]. The same group found in another study that the BCAA to aromatic amino acids (BCAA/AAA) ratio was significantly lower in sepsis patients compared to ICU controls [33]. The BCAA/AAA ratio is calculated in dividing (valine + leucine + isoleucine) by (phenylalanine + tyrosine) and is also called Fischer's ratio [41]. BCAA may enhance ammonia detoxification in muscles, and BCAA supplementation improves hepatic encephalopathy in liver cirrhosis [42,43]. The current guidelines for nutrition in the ICU do not mention BCAA, while nutritional guidelines recommend BCAA in liver cirrhosis patients $[44,45]$. In sepsis patients, those with septic encephalopathy were found to have a decreased BCAA/AAA ratio [46]. A study investigating different concentrations of BCAA in parenteral nutrition solutions found a higher BCAA content to be correlated with a more positive nitrogen balance and an increased BCAA/AAA ratio [29]. Data regarding mortality endpoints are not consistent, but studies in animals and humans found beneficial effects of BCAA rich nutrition [47-50]. We also assessed the BCAA/AAA ratio and found comparable results, but the prognostic value did not further increase compared to solely investigating BCAA. However, the AUROC curves in our study showed promising results and further improvement of the predictive value when dividing the SOFA score by BCAA. In addition, when assessing longitudinal data of sepsis patients, we found that non-survivors with lower BCAA levels died earlier than those having higher, but compared to survivors decreased, levels of BCAA.

The reason for a poor prognosis in sepsis patients with low BCAA levels is probably multifaceted. It may be partially driven by a pre-existing reduced muscle mass which leads to fewer reserves in critical illness, but as discussed before, it likely only accounts for a minor aspect. In addition, we cannot rule out alterations of enzymes such as the branched-chain alpha-keto acid dehydrogenase complex (BCKDC), which is a multienzyme located at the inner mitochondrial membrane. BCAA may be metabolized through an increased BCKDC activity during several forms of stress especially being present in septic shock [27,51]. Low BCAA levels may also constitute a surrogate parameter for gastrointestinal dysfunction leading to low uptake of essential amino acids in critically ill patients. Indeed, we found a correlation between SOFA score and BCAA, suggesting that the levels are decreased with increased organ dysfunction.

BCAA, with its anticatabolic effects, may provide a therapeutic target as shown in a study by Shirabe et al., where bacteremia rates were reduced in liver transplant recipients receiving oral BCAA supplements [52]. Studies have also shown beneficial effects of BCAA supplementation in several sarcopenic cohorts, including the elderly and patients with chronic liver disease comprising those with cirrhosis [53-58]. Furthermore, BCAA enhances muscle strength and mass [54,59-62]. A well-known risk factor for worse sepsis outcomes is sarcopenia [63-65]. Therefore, the supplementation of BCAA in sepsis patients may especially be essential in those with sarcopenia. Whether a BCAA supplementation containing all three amino acids or whether only a leucine supplementation may be beneficial in sepsis 
needs to be investigated in future studies [66]. In contrast to non-critically ill patients, the uptake from the intestine may be reduced in ICU patients, so a parenteral route leads to a higher bioavailability compared to oral supplementation.

The limitation of many previous metabolomic sepsis studies is the use of a targeted (i.e., known metabolites) approach, while in our study, an untargeted ${ }^{1} \mathrm{H}$ NMR technique was used for the investigation of metabolites. However, to allow for early prognosis prediction and to provide an early target for intervention, results of BCAA levels must be immediately available from central hospital laboratories, or close cooperation with metabolomic institutes with fast turn-around times is necessary. In our investigation, samples were extracted without lipoproteins to allow for the detection of small differences between groups, which may otherwise be lost to background noise. In general, data quality in the assessment of metabolites improves when lipoproteins are removed from samples. However, we additionally performed a targeted lipoprotein NMR approach and found altered levels of lipoproteins with lower HDL parameters in patients with sepsis compared to ICU controls. Still, lower quantitative lipoprotein levels were not associated with mortality outcomes. These results, analyzed with an independent method, confirm the findings of our previous study showing quantitative changes of lipoproteins to be less relevant than qualitative alterations [5].

Our study has several limitations: First, serum samples were used, and whole blood may provide even broader metabolomic results [67]. Second, we only included patients who were admitted to the ICU and earlier sampling points may be useful to predict the outcome. However, the presentation of patients within different times of the disease is a ubiquitous problem of sepsis studies. Furthermore, it must be noted that even an untargeted broad metabolomics approach is only a snapshot at a given time point. Third, another limitation of our study is sample size which limits external validity, and only the largest differences between groups may have been detected. Therefore, results need to be confirmed in larger studies. Fourth, we were not able to assess the type or amount of any recent oral food intake, as patients were critically ill, with many having an altered mental state on admission. Nevertheless, non-survivors had lower BCAA levels which may be used as a severity marker and may be investigated in future studies as a therapeutic target, independently of the history of food intake.

In conclusion, metabolomics using ${ }^{1} \mathrm{H}$ NMR spectroscopy is a promising field as part of a personalized medical approach providing robust and standardizable results, especially as sample preparation effort is low. Lipoproteins are significantly different in sepsis patients compared to ICU patients without sepsis or bacteremia. Furthermore, we were able to identify BCAA (valine, leucine, and isoleucine) as significantly different metabolites in sepsis survivors when compared to non-survivors, and these amino acids may be biomarkers for early outcome prediction. In the future, BCAA may be investigated as a target for therapeutic interventions.

Supplementary Materials: The following are available online at https:/ / www.mdpi.com/article/ 10.3390/nu13093106/s1, Table S1: Lipoprotein parameters in ICU sepsis patients and ICU controls, Table S2: Univariable logistic regression for lipoproteins (sepsis cohort), Table S3: Univariable logistic regression for lipoproteins (ICU controls), Table S4: Additional multivariable model for 28-day and ICU mortality including other BCAA, Table S5: Additional multivariable model for 28-day mortality including the SOFA score, Table S6: Baseline characteristics in longitudinal analyses.

Author Contributions: Conceptualization, A.C.R., G.M., H.S., and P.E.; data curation, A.C.R. and T.M.; formal analysis, A.C.R., F.P., and T.M.; funding acquisition, P.E.; investigation, A.C.R., G.M., B.B., and T.M.; project administration, P.E.; software, T.M.; supervision, G.M., H.S., and P.E.; visualization, A.C.R. and F.P.; writing-original draft, A.C.R., F.P., T.M., and P.E.; writing-review and editing, G.H., G.M., H.S., B.B., and K.E. All authors have read and agreed to the published version of the manuscript.

Funding: Doctoral student ACR received funding from the Medical University of Graz through the Doctoral School "Molecular Medicine and Inflammation" for article publication fees and open 
access costs. TM was supported by Austrian Science Fund (FWF) grants P28854, I3792, and DK-MCD W1226, the Austrian Research Promotion Agency (FFG) Grants 864690 and 870454; the Integrative Metabolism Research Center Graz; Austrian Infrastructure Program 2016/2017, the Styrian Government (Zukunftsfonds), and BioTechMed-Graz (Flagship project DYNIMO).

Institutional Review Board Statement: The study was conducted according to the guidelines of the Declaration of Helsinki, and approved by the Institutional Review Board of the Medical University of Graz (30-258 ex17/18, 06.04.2018).

Informed Consent Statement: Written informed consent was obtained from all conscious participants. In comatose non-survivors the local IRB acted as legal guardian and gave consent for participation within the study.

Data Availability Statement: The data presented in this study are available on request from the corresponding author.

Acknowledgments: We especially want to thank Anna-Maria Springer, BSc, who helped to prepare the samples for metabolomic analyses.

Conflicts of Interest: The authors declare no conflict of interest.

\section{References}

1. Fleischmann, C.; Scherag, A.; Adhikari, N.K.; Hartog, C.S.; Tsaganos, T.; Schlattmann, P.; Angus, D.C.; Reinhart, K.; International Forum of Acute Care, T. Assessment of Global Incidence and Mortality of Hospital-treated Sepsis. Current Estimates and Limitations. Am. J. Respir. Crit. Care Med. 2016, 193, 259-272. [CrossRef]

2. Gobatto, A.L.; Besen, B.A.; Azevedo, L.C. How Can We Estimate Sepsis Incidence and Mortality? Shock 2017, 47, 6-11. [CrossRef] [PubMed]

3. Singer, M.; Deutschman, C.S.; Seymour, C.W.; Shankar-Hari, M.; Annane, D.; Bauer, M.; Bellomo, R.; Bernard, G.R.; Chiche, J.D.; Coopersmith, C.M.; et al. The Third International Consensus Definitions for Sepsis and Septic Shock (Sepsis-3). JAMA 2016, 315, 801-810. [CrossRef]

4. Ryoo, S.M.; Han, K.S.; Ahn, S.; Shin, T.G.; Hwang, S.Y.; Chung, S.P.; Hwang, Y.J.; Park, Y.S.; Jo, Y.H.; Chang, H.L.; et al. The usefulness of C-reactive protein and procalcitonin to predict prognosis in septic shock patients: A multicenter prospective registry-based observational study. Sci. Rep. 2019, 9, 6579. [CrossRef]

5. Reisinger, A.C.; Schuller, M.; Holzer, M.; Stadler, J.T.; Hackl, G.; Posch, F.; Marsche, G.; Sourij, H.; Ekart, R.; Eller, K.; et al. Arylesterase Activity of HDL Associated Paraoxonase as a Potential Prognostic Marker in Patients With Sepsis and Septic Shock-A Prospective Pilot Study. Front. Med. (Lausanne) 2020, 7, 579677. [CrossRef] [PubMed]

6. Ludwig, K.R.; Hummon, A.B. Mass spectrometry for the discovery of biomarkers of sepsis. Mol. Biosyst. 2017, 13, 648-664. [CrossRef] [PubMed]

7. Everett, J.R. Pharmacometabonomics in humans: A new tool for personalized medicine. Pharmacogenomics 2015, 16, 737-754. [CrossRef]

8. Lee, J.; Banerjee, D. Metabolomics and the Microbiome as Biomarkers in Sepsis. Crit. Care Clin. 2020, 36, 105-113. [CrossRef] [PubMed]

9. Vignoli, A.; Ghini, V.; Meoni, G.; Licari, C.; Takis, P.G.; Tenori, L.; Turano, P.; Luchinat, C. High-Throughput Metabolomics by 1D NMR. Angew. Chem. Int. Ed. Engl. 2019, 58, 968-994. [CrossRef]

10. Alkan, H.F.; Walter, K.E.; Luengo, A.; Madreiter-Sokolowski, C.T.; Stryeck, S.; Lau, A.N.; Al-Zoughbi, W.; Lewis, C.A.; Thomas, C.J.; Hoefler, G.; et al. Cytosolic Aspartate Availability Determines Cell Survival When Glutamine Is Limiting. Cell Metab. 2018, 28, 706-720 e706. [CrossRef] [PubMed]

11. Chong, J.; Soufan, O.; Li, C.; Caraus, I.; Li, S.; Bourque, G.; Wishart, D.S.; Xia, J. MetaboAnalyst 4.0: Towards more transparent and integrative metabolomics analysis. Nucleic Acids Res. 2018, 46, W486-W494. [CrossRef]

12. Dieterle, F.; Ross, A.; Schlotterbeck, G.; Senn, H. Probabilistic quotient normalization as robust method to account for dilution of complex biological mixtures. Application in 1H NMR metabonomics. Anal. Chem. 2006, 78, 4281-4290. [CrossRef] [PubMed]

13. Huber, K.; Hofer, D.C.; Trefely, S.; Pelzmann, H.J.; Madreiter-Sokolowski, C.; Duta-Mare, M.; Schlager, S.; Trausinger, G.; Stryeck, S.; Graier, W.F.; et al. N-acetylaspartate pathway is nutrient responsive and coordinates lipid and energy metabolism in brown adipocytes. Biochim. Biophys. Acta Mol. Cell Res. 2019, 1866, 337-348. [CrossRef] [PubMed]

14. Maher, A.D.; Crockford, D.; Toft, H.; Malmodin, D.; Faber, J.H.; McCarthy, M.I.; Barrett, A.; Allen, M.; Walker, M.; Holmes, E.; et al. Optimization of human plasma 1H NMR spectroscopic data processing for high-throughput metabolic phenotyping studies and detection of insulin resistance related to type 2 diabetes. Anal. Chem. 2008, 80, 7354-7362. [CrossRef] [PubMed]

15. Vincent, J.L.; Jones, G.; David, S.; Olariu, E.; Cadwell, K.K. Frequency and mortality of septic shock in Europe and North America: A systematic review and meta-analysis. Crit. Care 2019, 23, 196. [CrossRef] 
16. Sakr, Y.; Jaschinski, U.; Wittebole, X.; Szakmany, T.; Lipman, J.; Namendys-Silva, S.A.; Martin-Loeches, I.; Leone, M.; Lupu, M.N.; Vincent, J.L.; et al. Sepsis in Intensive Care Unit Patients: Worldwide Data From the Intensive Care over Nations Audit. Open Forum Infect. Dis. 2018, 5, ofy313. [CrossRef]

17. Pierrakos, C.; Vincent, J.L. Sepsis biomarkers: A review. Crit. Care 2010, 14, R15. [CrossRef] [PubMed]

18. Evans, T. Diagnosis and management of sepsis. Clin. Med. (Lond.) 2018, 18, 146-149. [CrossRef]

19. Bonvini, A.; Coqueiro, A.Y.; Tirapegui, J.; Calder, P.C.; Rogero, M.M. Immunomodulatory role of branched-chain amino acids. Nutr. Rev. 2018, 76, 840-856. [CrossRef]

20. Li, P.; Yin, Y.L.; Li, D.; Kim, S.W.; Wu, G. Amino acids and immune function. Br. J. Nutr. 2007, 98, 237-252. [CrossRef]

21. Layman, D.K.; Walker, D.A. Potential importance of leucine in treatment of obesity and the metabolic syndrome. J. Nutr. 2006, 136, 319S-323S. [CrossRef]

22. Cummings, N.E.; Williams, E.M.; Kasza, I.; Konon, E.N.; Schaid, M.D.; Schmidt, B.A.; Poudel, C.; Sherman, D.S.; Yu, D.; Arriola Apelo, S.I.; et al. Restoration of metabolic health by decreased consumption of branched-chain amino acids. J. Physiol. 2018, 596, 623-645. [CrossRef]

23. Fontana, L.; Cummings, N.E.; Arriola Apelo, S.I.; Neuman, J.C.; Kasza, I.; Schmidt, B.A.; Cava, E.; Spelta, F.; Tosti, V.; Syed, F.A.; et al. Decreased Consumption of Branched-Chain Amino Acids Improves Metabolic Health. Cell Rep. 2016, 16, 520-530. [CrossRef] [PubMed]

24. Freund, H.R.; Ryan, J.A., Jr.; Fischer, J.E. Amino acid derangements in patients with sepsis: Treatment with branched chain amino acid rich infusions. Ann. Surg. 1978, 188, 423-430. [CrossRef]

25. Englert, J.A.; Rogers, A.J. Metabolism, Metabolomics, and Nutritional Support of Patients with Sepsis. Clin. Chest Med. 2016, 37, 321-331. [CrossRef] [PubMed]

26. Platell, C.; Kong, S.E.; McCauley, R.; Hall, J.C. Branched-chain amino acids. J. Gastroenterol. Hepatol. 2000, 15, 706-717. [CrossRef]

27. Holecek, M. Branched-chain amino acids in health and disease: Metabolism, alterations in blood plasma, and as supplements. Nutr. Metab. (Lond.) 2018, 15, 33. [CrossRef]

28. Sax, H.C.; Talamini, M.A.; Fischer, J.E. Clinical use of branched-chain amino acids in liver disease, sepsis, trauma, and burns. Arch. Surg. 1986, 121, 358-366. [CrossRef]

29. Jimenez Jimenez, F.J.; Ortiz Leyba, C.; Morales Menedez, S.; Barros Perez, M.; Munoz Garcia, J. Prospective study on the efficacy of branched-chain amino acids in septic patients. JPEN J. Parenter. Enteral Nutr. 1991, 15, 252-261. [CrossRef] [PubMed]

30. Asai, Y.; Bajotto, G.; Yoshizato, H.; Hamada, K.; Higuchi, T.; Shimomura, Y. The effects of endotoxin on plasma free amino acid concentrations in rats. J. Nutr. Sci. Vitaminol. (Tokyo) 2008, 54, 460-466. [CrossRef] [PubMed]

31. Lang, C.H.; Lynch, C.J.; Vary, T.C. BCATm deficiency ameliorates endotoxin-induced decrease in muscle protein synthesis and improves survival in septic mice. Am. J. Physiol. Regul. Integr. Comp. Physiol. 2010, 299, R935-R944. [CrossRef]

32. Druml, W.; Heinzel, G.; Kleinberger, G. Amino acid kinetics in patients with sepsis. Am. J. Clin. Nutr. 2001, 73, 908-913. [CrossRef]

33. Su, L.; Li, H.; Xie, A.; Liu, D.; Rao, W.; Lan, L.; Li, X.; Li, F.; Xiao, K.; Wang, H.; et al. Dynamic changes in amino acid concentration profiles in patients with sepsis. PLoS ONE 2015, 10, e0121933. [CrossRef]

34. Puskarich, M.A.; McHugh, C.; Flott, T.L.; Karnovsky, A.; Jones, A.E.; Stringer, K.A.; Investigators, R.T. Serum Levels of Branched Chain Amino Acids Predict Duration of Cardiovascular Organ Failure in Septic Shock. Shock 2021, 56, 65-72. [CrossRef]

35. Sprung, C.L.; Cerra, F.B.; Freund, H.R.; Schein, R.M.; Konstantinides, F.N.; Marcial, E.H.; Pena, M. Amino acid alterations and encephalopathy in the sepsis syndrome. Crit. Care Med. 1991, 19, 753-757. [CrossRef]

36. Liu, Z.; Triba, M.N.; Amathieu, R.; Lin, X.; Bouchemal, N.; Hantz, E.; Le Moyec, L.; Savarin, P. Nuclear magnetic resonance-based serum metabolomic analysis reveals different disease evolution profiles between septic shock survivors and non-survivors. Crit. Care 2019, 23, 169. [CrossRef]

37. Mickiewicz, B.; Duggan, G.E.; Winston, B.W.; Doig, C.; Kubes, P.; Vogel, H.J.; Alberta Sepsis, N. Metabolic profiling of serum samples by $1 \mathrm{H}$ nuclear magnetic resonance spectroscopy as a potential diagnostic approach for septic shock. Crit. Care Med. 2014, 42, 1140-1149. [CrossRef] [PubMed]

38. Mickiewicz, B.; Tam, P.; Jenne, C.N.; Leger, C.; Wong, J.; Winston, B.W.; Doig, C.; Kubes, P.; Vogel, H.J.; Alberta Sepsis, N. Integration of metabolic and inflammatory mediator profiles as a potential prognostic approach for septic shock in the intensive care unit. Crit. Care 2015, 19, 11. [CrossRef]

39. Blaise, B.J.; Gouel-Cheron, A.; Floccard, B.; Monneret, G.; Allaouchiche, B. Metabolic phenotyping of traumatized patients reveals a susceptibility to sepsis. Anal. Chem. 2013, 85, 10850-10855. [CrossRef] [PubMed]

40. Su, L.; Huang, Y.; Zhu, Y.; Xia, L.; Wang, R.; Xiao, K.; Wang, H.; Yan, P.; Wen, B.; Cao, L.; et al. Discrimination of sepsis stage metabolic profiles with an LC/MS-MS-based metabolomics approach. BMJ Open Respir. Res. 2014, 1, e000056. [CrossRef]

41. Fischer, J.E.; Rosen, H.M.; Ebeid, A.M.; James, J.H.; Keane, J.M.; Soeters, P.B. The effect of normalization of plasma amino acids on hepatic encephalopathy in man. Surgery 1976, 80, 77-91. [PubMed]

42. Gluud, L.L.; Dam, G.; Les, I.; Marchesini, G.; Borre, M.; Aagaard, N.K.; Vilstrup, H. Branched-chain amino acids for people with hepatic encephalopathy. Cochrane Database Syst. Rev. 2017, 5, CD001939. [CrossRef] [PubMed]

43. Dam, G.; Aamann, L.; Vistrup, H.; Gluud, L.L. The role of Branched Chain Amino Acids in the treatment of hepatic Encephalopathy. J. Clin. Exp. Hepatol. 2018, 8, 448-451. [CrossRef] [PubMed]

44. Plauth, M.; Bernal, W.; Dasarathy, S.; Merli, M.; Plank, L.D.; Schutz, T.; Bischoff, S.C. ESPEN guideline on clinical nutrition in liver disease. Clin. Nutr. (Edinb. Scotl.) 2019, 38, 485-521. [CrossRef] [PubMed] 
45. Singer, P.; Blaser, A.R.; Berger, M.M.; Alhazzani, W.; Calder, P.C.; Casaer, M.P.; Hiesmayr, M.; Mayer, K.; Montejo, J.C.; Pichard, C.; et al. ESPEN guideline on clinical nutrition in the intensive care unit. Clin. Nutr. (Edinb. Scotl.) 2019, 38, 48-79. [CrossRef]

46. Basler, T.; Meier-Hellmann, A.; Bredle, D.; Reinhart, K. Amino acid imbalance early in septic encephalopathy. Intensive Care Med. 2002, 28, 293-298. [CrossRef]

47. Garcia-de-Lorenzo, A.; Ortiz-Leyba, C.; Planas, M.; Montejo, J.C.; Nunez, R.; Ordonez, F.J.; Aragon, C.; Jimenez, F.J. Parenteral administration of different amounts of branch-chain amino acids in septic patients: Clinical and metabolic aspects. Crit. Care Med. 1997, 25, 418-424. [CrossRef]

48. Mori, E.; Hasebe, M.; Kobayashi, K. Effect of total parenteral nutrition enriched in branched-chain amino acids on metabolite levels in septic rats. Metab. Clin. Exp. 1988, 37, 824-830. [CrossRef]

49. Fujita, H.; Hirose, K.; Miyazaki, I. Effects of branched chain enriched amino acid solutions on septic rats. Clin. Nutr. (Edinb. Scotl.) 1986, 5, 171-177. [CrossRef]

50. Vente, J.P.; Soeters, P.B.; von Meyenfeldt, M.F.; Rouflart, M.M.; van der Linden, C.J.; Gouma, D.J. Prospective randomized double-blind trial of branched chain amino acid enriched versus standard parenteral nutrition solutions in traumatized and septic patients. World J. Surg. 1991, 15, 128-132, discussion 133. [CrossRef]

51. Nicastro, H.; da Luz, C.R.; Chaves, D.F.; Bechara, L.R.; Voltarelli, V.A.; Rogero, M.M.; Lancha, A.H., Jr. Does Branched-Chain Amino Acids Supplementation Modulate Skeletal Muscle Remodeling through Inflammation Modulation? Possible Mechanisms of Action. J. Nutr. Metab. 2012, 2012, 136937. [CrossRef]

52. Shirabe, K.; Yoshimatsu, M.; Motomura, T.; Takeishi, K.; Toshima, T.; Muto, J.; Matono, R.; Taketomi, A.; Uchiyama, H.; Maehara, Y. Beneficial effects of supplementation with branched-chain amino acids on postoperative bacteremia in living donor liver transplant recipients. Liver Transpl. 2011, 17, 1073-1080. [CrossRef]

53. Hey, P.; Gow, P.; Testro, A.G.; Apostolov, R.; Chapman, B.; Sinclair, M. Nutraceuticals for the treatment of sarcopenia in chronic liver disease. Clin. Nutr. ESPEN 2021, 41, 13-22. [CrossRef] [PubMed]

54. Hernandez-Conde, M.; Llop, E.; Gomez-Pimpollo, L.; Fernandez Carrillo, C.; Rodriguez, L.; Van Den Brule, E.; Perello, C.; Lopez-Gomez, M.; Abad, J.; Martinez-Porras, J.L.; et al. Adding Branched-Chain Amino Acids to an Enhanced Standard-of-Care Treatment Improves Muscle Mass of Cirrhotic Patients With Sarcopenia: A Placebo-Controlled Trial. Am. J. Gastroenterol. 2021. [CrossRef]

55. Ko, C.H.; Wu, S.J.; Wang, S.T.; Chang, Y.F.; Chang, C.S.; Kuan, T.S.; Chuang, H.Y.; Chang, C.M.; Chou, W.; Wu, C.H. Effects of enriched branched-chain amino acid supplementation on sarcopenia. Aging (Albany NY) 2020, 12, 15091-15103. [CrossRef]

56. Hanai, T.; Shiraki, M.; Nishimura, K.; Ohnishi, S.; Imai, K.; Suetsugu, A.; Takai, K.; Shimizu, M.; Moriwaki, H. Sarcopenia impairs prognosis of patients with liver cirrhosis. Nutrition 2015, 31, 193-199. [CrossRef]

57. Vasques, J.; Guerreiro, C.S.; Sousa, J.; Pinto, M.; Cortez-Pinto, H. Nutritional support in cirrhotic patients with sarcopenia. Clin. Nutr. ESPEN 2019, 33, 12-17. [CrossRef]

58. de Campos-Ferraz, P.L.; Andrade, I.; das Neves, W.; Hangai, I.; Alves, C.R.; Lancha, A.H., Jr. An overview of amines as nutritional supplements to counteract cancer cachexia. J. Cachexia Sarcopenia Muscle 2014, 5, 105-110. [CrossRef]

59. Fedewa, M.V.; Spencer, S.O.; Williams, T.D.; Becker, Z.E.; Fuqua, C.A. Effect of branched-Chain Amino Acid Supplementation on Muscle Soreness following Exercise: A Meta-Analysis. Int. J. Vitam. Nutr. Res. 2019, 89, 348-356. [CrossRef]

60. Park, S.; Chae, M.; Park, H.; Park, K. Higher Branched-Chain Amino Acid Intake Is Associated with Handgrip Strength among Korean Older Adults. Nutrients 2021, 13, 1522. [CrossRef] [PubMed]

61. Waldron, M.; Whelan, K.; Jeffries, O.; Burt, D.; Howe, L.; Patterson, S.D. The effects of acute branched-chain amino acid supplementation on recovery from a single bout of hypertrophy exercise in resistance-trained athletes. Appl. Physiol. Nutr. Metab. 2017, 42, 630-636. [CrossRef] [PubMed]

62. VanDusseldorp, T.A.; Escobar, K.A.; Johnson, K.E.; Stratton, M.T.; Moriarty, T.; Cole, N.; McCormick, J.J.; Kerksick, C.M.; Vaughan, R.A.; Dokladny, K.; et al. Effect of Branched-Chain Amino Acid Supplementation on Recovery Following Acute Eccentric Exercise. Nutrients 2018, 10, 1389. [CrossRef] [PubMed]

63. Ji, Y.; Cheng, B.; Xu, Z.; Ye, H.; Lu, W.; Luo, X.; Fu, S.; Fang, X. Impact of sarcopenic obesity on 30-day mortality in critically ill patients with intra-abdominal sepsis. J. Crit. Care 2018, 46, 50-54. [CrossRef]

64. Loosen, S.H.; Schulze-Hagen, M.; Pungel, T.; Bundgens, L.; Wirtz, T.; Kather, J.N.; Vucur, M.; Paffenholz, P.; Demir, M.; Bruners, P.; et al. Skeletal Muscle Composition Predicts Outcome in Critically Ill Patients. Crit. Care Explor. 2020, 2 , e0171. [CrossRef]

65. Cox, M.C.; Booth, M.; Ghita, G.; Wang, Z.; Gardner, A.; Hawkins, R.B.; Darden, D.B.; Leeuwenburgh, C.; Moldawer, L.L.; Moore, F.A.; et al. The impact of sarcopenia and acute muscle mass loss on long-term outcomes in critically ill patients with intra-abdominal sepsis. J. Cachexia Sarcopenia Muscle 2021. [CrossRef] [PubMed]

66. De Bandt, J.P.; Cynober, L. Therapeutic use of branched-chain amino acids in burn, trauma, and sepsis. J. Nutr. 2006, 136, 308S-313S. [CrossRef]

67. Stringer, K.A.; Younger, J.G.; McHugh, C.; Yeomans, L.; Finkel, M.A.; Puskarich, M.A.; Jones, A.E.; Trexel, J.; Karnovsky, A. Whole Blood Reveals More Metabolic Detail of the Human Metabolome than Serum as Measured by 1H-NMR Spectroscopy: Implications for Sepsis Metabolomics. Shock 2015, 44, 200-208. [CrossRef] 